\title{
ON THE INFLUENCE OF BORIC ACID AND BORAX UPON THE GENERAL METABOLISM OF CHILDREN.
}

\author{
By F. W. TUNNICLIFFE, M.D., M.R.C.P., \\ Professor of Materia Medica and Pharmacology in King's College, London; \\ and OTTO ROSENheIM, Ph.D., F.C.S.
}

Bотн boric acid and borax are extensively used as food preservatives, and much interest attaches to the question of their influence if any upon the general nutrition of the consumer, especially children. Although this method of preserving food has existed for a comparatively long period there seeins no agreement as to the harmfulness or otherwise of these substances when taken with a mixed diet.

The method most valuable for affording us reliable data upon which to base conclusions in this connection is that of comparative metabolic observations on the human subject, extending over a considerable period, and this method we have adopted.

Before entering fully into our own work in this direction we shall briefly criticise the data from which up to the present the conclusions concerning the harmfulness or otherwise of boric acid and borax as food preservatives have been based.

Literature. The literature may be summarised as consisting of, (I.) Experiments made on the influence of these substances upon digestions in vitro, (II.) Experiments made on animals as to the effect of prolonged small doses upon their general health and metabolism, (III.) General action on man and one metabolic experiment made on one man. 


\section{Experiments made upon the infuence of boric acid and borax upon digestions in vitro.}

Comparative qualitative experiments have been carried out in this connection by Hehner ${ }^{1}$, Weber ${ }^{2}$, F. J. Allen ${ }^{3}$, Cripps ${ }^{4}$, Leffmann ${ }^{5}$, Liebreich $^{6}$, Halliburton ${ }^{7}$ Quantitative experiments have also been made by Chittenden ${ }^{8}$, Maybery and Goldsmith ${ }^{9}$, Rideal and Foulerton ${ }^{10}$, and Liebreich ${ }^{11}$.

The outcome of the quantitative experiments which confirm the qualitative ones may shortly be summarised as follows:

Salivary Digestion: Boric acid favours the amylolytic action of saliva (Chittenden). Borax on the other hand has an inhibitory action on the conversion of starch by saliva (Weber, Chittenden, Rideal and Foulerton, Liebreich). This latter effect is shown by Liebreich to be an alkali-action.

Rennet Action: Boric acid either has no influence upon the action of rennet upon milk (Cripps, Halliburton) or hastens it (F. J. Allen). Borax, according to the concentration, delays or prevents rennet action (F. J. Allen, Halliburton). By the addition of small quantities of calcium chloride, however, the rennet action takes place in the presence of borax (Allen). It is interesting in this connection to note that sodium chloride has the same action as borax ${ }^{12}$.

Gastric Digestion: Boric acid in large doses favours gastric proteolysis $^{13}$ (Chittenden). Borax in small doses has also a slight accelerating action (Chittenden, Rideal and Foulerton), whilst in large doses according to the increasing alkalinity it has a retarding effect (Chittenden).

Pancreatic Digestion: (a) Proteolysis. Borax in small and large doses, proportionally to its concentration, stimulates markedly pancreatic proteolysis (Chittenden). Boric acid (and boric mixture) have

1 Analyst, 1891, p. 126.

3 Lancet, 1896 (I.), p. 1516.

2 Journ. Americ. Chem. Soc., 1892, p. 4.

5 Journ. Franklin Inst., 1899, p. 103.

6 Vierteljahrsschr. f. gerichtl. Medicin, 1900, p. 83.

7 Brit. Med. Journ., 1900, II. p. 1.

8 Dietetic and Hygienic Gazette, 1893, p. 25.

9 Journ. Americ. Chem. Soc., 1897, p. 889.

10 Public Health, 1899, No. 3, p. 554.

12 Ringer, Journ. of Physiol. 1895, p. 425.

13 Maybery and Goldsmith's results, apparently in conflict with the above statement, are however vitiated by the variation in their control experiments.

Journ. of Hyg. I 
a distinct inhibitory action. (b) Amylolysis. Borax mixture exerts a retarding action on the conversion of starch by commercial pancreatic extract (Rideal and Foulerton). Borax itself has a slight retarding action, whilst boric acid has no action (Liebreich).

The results of the above observers seem to justify the conclusion that (the radicle of) boric acid and borax as such exerts no specific action, the effect in each case being referable to the acid, or alkali moiety, all digestions taking place in an acid medium being inhibited by borax, those occurring in an alkaline medium by boric acid.

\section{Experiments made upon animals.}

(a) Effect of prolonged small doses. Animals have been fed for different periods with food containing various quantities of borax and boric acid by several observers (Neumann ${ }^{1}$, Annett $^{2}$, Rideal ${ }^{3}$, Liebreich ${ }^{4}$ ). The outcome of these experiments is shortly that boric acid and borax given in small doses for prolonged periods have no influence on the general health of animals (Neumann, Rideal, Liebreich).

Excessive doses (10 grammes or more according to body weight) produced transient nausea and vomiting.

To elucidate the question of the effect of boric acid upon young animals ${ }^{5}$ a series of experiments were made upon young sucking-pigs by A. D. Hall and H. S. Hammond in collaboration with ourselves at the South-Eastern Agricultural College, Wye. These observations ${ }^{6}$ show that 0.2 to 2.4 grammes boric acid per diem continued for seven weeks added to a mixed weighed diet had no influence upon the live weight, growth, and general health of the animals.

(b) Metabolic experiments on animals have been made by $\mathrm{Cyon}^{7}$, Gruber $^{8}$, Chittenden and Gies ${ }^{9}$, and Liebreich ${ }^{10}$.

Chittenden and Gies' experiments are very complete and accurate, and in their paper will be found a detailed criticism of the earlier less complete work. The chief conclusions from Chittenden and Gies' work are best given in their own words shortly as follows:

1 Arch. f. exp. Path. u. Pharm. 1881, p. $149 . \quad 2$ Lancet, 1899, II. p. 1282.

3 Lancet, 1900, r. p. 228.

4 loc. cit.

5 The experiments of Annett (loc. cit.) in this regard cannot be considered conclusive. Vide Liebreich (Lancet, 1900, I. p. 13), and Rideal (loc. cit.).

6 These observations will be published in extenso elsewhere.

7 Comptes rendus, 1878, T. LxxxvIr. p. 845.

8 Zeitschr. f. Biologie, 1880, p. 198.

9 American Journ. of Physiol. 1898, p. $1 . \quad{ }^{10}$ loc. cit. 
Moderate doses of borax up to 5 grammes per day, when continued for some time, are without influence upon proteid metabolism and do not exert any influence upon the general nutritional changes of the body. Large doses of borax, 5-10 grammes daily, have a direct stimulating effect upon proteid metabolism. They tend to retard somewhat the assimilation of proteid and fatty foods, increasing notably the weight of the faeces and their contents of nitrogen and fat.

Boric acid in doses up to 3 grammes per day is practically without influence upon proteid metabolism and general nutrition.

Neither boric acid nor borax affects intestinal putrefaction.

Liebreich's experiment with borax on a dog confirmed Chittenden and Gies' results.

\section{General action on man, etc.}

There is abundant evidence that boric acid and borax can be taken by man in considerable doses over long periods in the food, or by itself without producing any toxic effect ${ }^{1}$. It is also, however, definitely established that in certain patients medicinal doses ( 1 gramme two or three times a day) give rise to transient erythematous eruptions after relatively short periods. It is, however, to be noted that these eruptions were, so far as we are aware, invariably produced by the use of boric acid or borax as a drug, no case being reported of the occurrence of rashes caused by these substances in food. How far these rashes depend on idiosyncrasy and are comparable to those produced by shellfish, strawberries, etc., is outside our purpose to discuss here.

The only metabolic experiment made on man published up till now is one by Forster ${ }^{2}$. The action of boric acid alone on one man was examined. Two relatively short series of experiments were made. In the first 3 grammes of boric acid were given daily for three days with a mixed diet. The second experiment consisted of two periods of two days each in which the subject, an adult man, took 1.5 and 0.5 grammes of boric acid per diem respectively with a milk diet. Forster's conclusions, which appear to have been much more cautiously expressed by himself than by his abstractors, are that boric acid has no influence on proteid metabolism and fat assimilation. He found,

1 R. Virchow, Berl. klin. Wochenschr. No. 1, 1884 ; Gaucher, Bull. Méd., 1890, No. 46 (quoted from Lehmann, Die Methoden der prakt. Hygiene, Wiesbaden, 1901, p. 305), amongst many others.

2 Arch. f. Hygiene, 1884, ir. p. 75. 
however, that the total quantity of faeces and their nitrogen and phosphorus percentage were slightly increased. Forster is inclined to ascribe this to a slightly diminished assimilation, together with an increased epithelial and mucous excretion from the intestine. (This latter, however, he regards as being purely hypothetical.) $\mathrm{He}$ also concludes that boric acid may possibly exert an intestinal antiseptic action, as indicated by the slight decrease of the ethereal sulphates in the urine.

Lehmann in his Methoden der prakt. Hygiene, 1901, states that the results of an observation made under his direction by $\mathrm{K}$. Mann upon the latter's own metabolism (not yet published) did not confirm those of Forster.

\section{General aRRANGEMENT AND METHOD OF OUR OBSERVATIONS.}

Before entering into the details especially relevant to our own observations it might be well to recapitulate briefly the general principles of metabolic experiments. They consist in the exact estimation of the quantity of food and its various constituents during a given period, and the estimation during the same period of the total excreta and their constituents, chiefly with regard to Nitrogen, Phosphorus, Fat, etc. By this means we get valuable information with regard to the assimilation of these substances by and their retention in the body. We should like to point out however that there is a slight fallacy in this reasoning, in that our knowledge of the origin of faecal nitrogen is somewhat limited. According to Prausnitz ${ }^{1}$ the whole of the nitrogen in the faeces arises not from the unabsorbed nitrogen of the food, but from the intestinal secretion (epithelial cells, etc.). The value of the metabolic method is not to any extent affected by this, in so far as all nitrogen excreted by the faeces must be regarded as lost to the body, and its subtraction from the quantity of nitrogen ingested gives us the quantity of nitrogen retained. It must, however, be observed at once as has been emphasised by Pawlow ${ }^{2}$ that the results of these experiments give us no absolute information with regard to the actual digestibility of any given food, in so far as we are left by them in complete ignorance of the amount of energy spent by the organism in producing the observed effect. Provided the organism is equal to the occasion an indigestible food might be as

1 Zeitschr. f. Biologie, 1897, p. 287.

${ }^{2}$ Die Arbeit der Verduaungsdrïsen, Wiesbaden, 1898. 
well assimilated and retained as a digestible one, but to produce this result an additional output of energy would be required. If this additional output of energy were relatively small we should probably have no indications with regard to it, but were it relatively large, or in other words were the difference in the digestibility of the foods in question great, we should probably find that the body weight or the general health of the person under observation would be affected, and this effect would be the more noticeable the longer the period of observation, and the more sensitive the person chosen. From these reasons we thought it advisable to allow our observations on general principles to extend over comparatively long periods, and to take what were a priori to be regarded as relatively sensitive reagents, viz. children both robust and delicate, and to observe minutely during the various periods their general health and behaviour. From another standpoint children had, in this connection, an additional interest, on account of the fact that milk forms so large a proportion of their diet, and it is to milk that boric acid and borax as preservatives are generally added.

Our observations were made upon three children, two of whom (boys) might be regarded as typically healthy, and were aged $2 \frac{1}{2}$ and 5 years, the third child (girl aged 4 years) was delicate, being convalescent from pneumonia. We shall refer to the children subsequently as $A, B$, and $C$, respectively. During the whole period the children were under our perpetual observation, and absolute control was kept over all ingesta, which were accurately weighed by us, and excreta, which were collected in diurnal periods without loss. The general conditions of their life remained constant, they were kept for some time before the "fore period" of the observation began under identical conditions to those obtaining during the observation, they took each day the same amount of exercise, and their habits were in every respect regular. The research was carried out during the months of May and June, and extended in the case of $B$ and $C$ over a period of 22 days, in the case of $A$ over one of 25 days. Each period was subdivided into four, a fore, a boric acid, a borax, and an after period. The relative lengths of these periods will be seen from the tables. The children had a mixed diet. With regard to the quantities of the different food stuffs we were guided at first by the work of Camerer ${ }^{1}$. This was subsequently modified to a small extent by our own observations concerning the establishment of nitrogenous equilibrium in which

1 Der Stoffwechsel des Kindes. Tübingen, 1896. 
the children were approximately placed before the fore period began. Every article of food was carefully analysed, with regard to its percentage composition, and in no case were so called average figures taken.

In order to minimise the amount of analytical work entailed by this method the three children were supplied from the same stock of foods, which were taken originally in as large a quantity as was consistent with their keeping properties. To this end pasteurized milk was supplied to us in bottles, each lot of bottles being taken from the same churn ${ }^{1}$. Each lot of meat lasted for about four days, lean beef was usually taken, and the whole stock minced, a sample of this was then analysed.

The following table shows the percentage composition of the foods used :-

\section{TABLE I.}

Showing the Percentage Composition of the Foods.

\begin{tabular}{|c|c|c|c|c|c|c|c|c|}
\hline & & $\begin{array}{l}\text { Specific } \\
\text { gravity }\end{array}$ & $\underset{\% \%}{\text { Water }}$ & Fat & $\begin{array}{c}\text { Total } \\
\text { carbohydrates } \\
\% \%\end{array}$ & $\begin{array}{c}\text { Nitrogen } \\
\%\end{array}$ & $\begin{array}{l}\text { Phosphoric } \\
\text { acid } \\
\%\end{array}$ & $\begin{array}{c}\text { Ash } \\
0 \%\end{array}$ \\
\hline 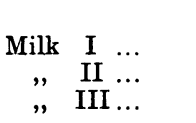 & $\begin{array}{l}\cdots \\
\cdots \\
\cdots\end{array}$ & $\begin{array}{l}1 \cdot 0310 \\
1 \cdot 0310 \\
1 \cdot 0330\end{array}$ & $\begin{array}{l}88 \cdot 65 \\
86 \cdot 63 \\
87 \cdot 61\end{array}$ & $\begin{array}{l}3 \cdot 00 \\
4 \cdot 20 \\
3 \cdot 10\end{array}$ & $\begin{array}{c}\text { Lactose } \\
4 \cdot 61 \\
4 \cdot 60 \\
4 \cdot 94\end{array}$ & $\begin{array}{l}0.52 \\
0.53 \\
0.56\end{array}$ & $\begin{array}{l}0 \cdot 23 \\
0 \cdot 27 \\
0 \cdot 27\end{array}$ & $\begin{array}{l}0 \cdot 69 \\
0 \cdot 69 \\
0 \cdot 72\end{array}$ \\
\hline $\begin{array}{cc}\text { Bread } & \text { I } \\
, " & \text { II } \\
, & \text { III }\end{array}$ & $\begin{array}{l}\cdots \\
\cdots \\
\cdots\end{array}$ & - & $\begin{array}{l}37 \cdot 90 \\
37 \cdot 90 \\
36 \cdot 90\end{array}$ & $\begin{array}{l}0 \cdot 14 \\
0 \cdot 13 \\
0 \cdot 18\end{array}$ & $\begin{array}{c}\text { Dextrose } \\
55 \cdot 97 \\
55 \cdot 97 \\
58.02\end{array}$ & $\begin{array}{l}1 \cdot 20 \\
1 \cdot 20 \\
1 \cdot 13\end{array}$ & $\begin{array}{l}0 \cdot 16 \\
0 \cdot 16 \\
0 \cdot 15\end{array}$ & $\begin{array}{l}0.79 \\
0.79 \\
0.48\end{array}$ \\
\hline $\begin{array}{cc}\text { Butter } & \text { I } \\
, " & \text { II } \\
, & \text { III }\end{array}$ & $\begin{array}{l}\cdots \\
\cdots \\
\cdots\end{array}$ & - & $\begin{array}{l}12 \cdot 68 \\
14 \cdot 56 \\
12 \cdot 43\end{array}$ & $\begin{array}{l}86 \cdot 00 \\
84 \cdot 37 \\
85 \cdot 69\end{array}$ & $\begin{array}{c}\text { Lactose } \\
0.14 \\
0.29 \\
0.16\end{array}$ & $\begin{array}{l}0 \cdot 11 \\
0.08 \\
0 \cdot 19\end{array}$ & - & $\begin{array}{l}0.50 \\
0.29 \\
0.51\end{array}$ \\
\hline $\begin{array}{cc}\text { Meat } & \text { I } \ldots . \\
, & \text { II } \ldots \\
, & \text { III ... } \\
, & \text { IV ... }\end{array}$ & $\begin{array}{l}\cdots \\
\cdots \\
\cdots \\
\cdots\end{array}$ & $\begin{array}{l}- \\
-\end{array}$ & $\begin{array}{l}72 \cdot 85 \\
69 \cdot 22 \\
73 \cdot 70 \\
73 \cdot 91\end{array}$ & $\begin{array}{r}2 \cdot 58 \\
10 \cdot 23 \\
2 \cdot 93 \\
2 \cdot 74\end{array}$ & $\begin{array}{c}\text { Dextrose } \\
- \\
- \\
-\end{array}$ & $\begin{array}{l}3 \cdot 88 \\
3 \cdot 12 \\
3 \cdot 33 \\
3 \cdot 34\end{array}$ & $\begin{array}{l}0.45 \\
0.42 \\
0.43 \\
0.39\end{array}$ & $\begin{array}{l}1 \cdot 16 \\
1 \cdot 08 \\
1 \cdot 17 \\
1 \cdot 14\end{array}$ \\
\hline $\begin{array}{c}\text { Apple Compote } \\
", "\end{array}$ & $\underset{\text { III }}{\text { I }}$ & 二 & $\begin{array}{l}63 \cdot 02 \\
75 \cdot 80 \\
63 \cdot 18\end{array}$ & E & $\begin{array}{c}\text { Dextrose } \\
29 \cdot 84 \\
21 \cdot 80 \\
31 \cdot 08\end{array}$ & $\begin{array}{l}0.06 \\
0.05 \\
0.13\end{array}$ & $\begin{array}{l}0.04 \\
0.04 \\
0.06\end{array}$ & $\begin{array}{l}0 \cdot 33 \\
0 \cdot 34 \\
0 \cdot 43\end{array}$ \\
\hline Toffee $\quad \ldots$ & $\ldots$ & - & $3 \cdot 14$ & $4 \cdot 33$ & $\begin{array}{c}\text { Dextrose } \\
76.95\end{array}$ & 0.03 & - & - \\
\hline
\end{tabular}

1 For this we are indebted to Mr Droop Richmond of the Aylesbury Dairy Co. 
The excreta were collected without loss in twenty-four hour periods, from $8 \mathrm{a} . \mathrm{m}$. to $8 \mathrm{a} . \mathrm{m}$., and worked up the same day. The faeces were weighed in their normal state each day, small quantities of acid added when necessary, and subsequently evaporated on a water-bath. When dry they were finely powdered and analysed. The faeces belonging to each period were separated by means of the administration of powdered charcoal.

Methods of Analysis. All nitrogen estimations were made by Gunning's ${ }^{1}$ modification of Kjeldahl's method. It was found advantageous, especially in the analysis of the faeces, to add a few crystals of copper sulphate to the mixture of sulphuric acid and potassium sulphate, as by this means a very rapid and quiet oxidation was obtained. Two methods of phosphorus estimation were used. In food, faeces, and urine, the total phosphorus was estimated by Neumann-Keller's method ${ }^{2}$, viz. by oxidation in a Kjeldahl's flask by means of nitric acid and ammonium nitrate, subsequent precipitation with molybdic solution etc., and weighing as magnesium pyrophosphate. For the estimation of lecithin phosphorus in the ethereal extract of the faeces the usual process was used, oxidation by means of a mixture of sodium carbonate and nitrate and subsequent estimation of the phosphorus as before. The carbohydrates were estimated gravimetrically as dextrose or lactose by means of Fehling's solution. The fats, which term includes all the ether soluble substances, were estimated by extraction in Soxhlet's apparatus, after previous treatment with alcohol according to E. V. Voit's. Lecithin was estimated by multiplying the phosphorus figure obtained from the filtered ethereal extract of the faeces with the factor $7 \cdot 27$, corresponding to distearyl-lecithin. The uric acid was determined by our own modification of Hopkins' method ${ }^{4}$. The total and ethereal sulphuric acids were estimated according to Baumann's method ${ }^{5}$.

1 Zeitschr. für analyt. Chemie, 1889, p. 89.

2 Zeitschr. für physiol. Chemie, xxIx. p. 151.

3 Zeitschr. für Biol., xxvir. p. 555.

4 Centralbl. $f$. Physiol., 1897, p. 434. It has been shown by the most recent workers that the initial precipitation of uric acid by means of ammonium chloride is just as reliable as the more complicated method of Salkowski-Ludwig when certain conditions are observed. Ritter, Folin, Wörner, etc.

5 Zeitschr. f. physiol. Chem., r. 70. See also Neubauer and Vogel, Analyse des Harns, p. 724 . 
176 Influence of Boric Acid and Borax, etc.

TABLE II.

Showing the Influence of Boric Acid and Borax

\begin{tabular}{|c|c|c|c|c|c|c|c|c|c|c|}
\hline \multirow[b]{2}{*}{ PERIOD } & \multirow[b]{2}{*}{-} & \multirow[b]{2}{*}{ Date } & \multirow[b]{2}{*}{$\begin{array}{c}\text { Dose } \\
\mathrm{g} \\
\end{array}$} & \multicolumn{7}{|c|}{ URINE } \\
\hline & & & & $\begin{array}{c}\text { Quantity } \\
\text { c.c. }\end{array}$ & Reaction & $\begin{array}{l}\text { Specific } \\
\text { gravity }\end{array}$ & $\begin{array}{c}\text { Total } \\
\text { sulphuric } \\
\text { acid } \\
\text { g }\end{array}$ & $\begin{array}{c}\text { Ethereal } \\
\text { sulphuric } \\
\text { acid } \\
\mathbf{g}\end{array}$ & $\begin{array}{c}\text { Uric } \\
\text { acid } \\
\text { g }\end{array}$ & $\begin{array}{c}\text { Nitrogen } \\
\mathrm{g}\end{array}$ \\
\hline \multirow[t]{2}{*}{$\begin{array}{l}\text { FORE } \\
\text { PERIOD }\end{array}$} & & $\begin{array}{l}9 \mathrm{~V} \\
10, \\
11, \\
12, \\
13, \\
14, \\
15, \\
16,\end{array}$ & - & $\begin{array}{l}240 \\
310 \\
345 \\
445 \\
440 \\
325 \\
285 \\
245\end{array}$ & $\begin{array}{c}\text { Amphoteric } \\
\text { Acid } \\
\text { Amphoteric } \\
\text { Acid } \\
\text { Amphoteric } \\
\text {," }\end{array}$ & $\begin{array}{l}1 \cdot 0290 \\
1 \cdot 0268 \\
1 \cdot 0230 \\
1 \cdot 0195 \\
1 \cdot 0180 \\
1 \cdot 0235 \\
1 \cdot 0226 \\
1 \cdot 0238\end{array}$ & $\begin{array}{l}0 \cdot 6845 \\
0 \cdot 8841 \\
0.9839 \\
1 \cdot 2691 \\
1 \cdot 2551 \\
0 \cdot 9373 \\
0 \cdot 8220 \\
0 \cdot 7066\end{array}$ & $\begin{array}{l}0.0322 \\
0.0415 \\
0.0462 \\
0.0596 \\
0.0590 \\
0.0442 \\
0.0388 \\
0.0333\end{array}$ & $\begin{array}{l}0 \cdot 1350 \\
0 \cdot 1744 \\
0 \cdot 1941 \\
0 \cdot 2503 \\
0 \cdot 2475 \\
0 \cdot 0683 \\
0 \cdot 0999 \\
0 \cdot 0515\end{array}$ & $\begin{array}{l}4 \cdot 52 \\
5 \cdot 42 \\
5 \cdot 15 \\
5 \cdot 59 \\
5 \cdot 13 \\
4 \cdot 95 \\
4 \cdot 21 \\
4 \cdot 11\end{array}$ \\
\hline & $\begin{array}{l}\text { Total } \\
\text { Average }\end{array}$ & $\begin{array}{l}8 \text { days } \\
1 \text { day }\end{array}$ & & $\begin{array}{r}2,635 \\
325\end{array}$ & & $1 \cdot 0233$ & $\begin{array}{l}7 \cdot 5426 \\
0 \cdot 9428\end{array}$ & \begin{tabular}{|l|}
0.3548 \\
0.0444
\end{tabular} & $\begin{array}{l}1 \cdot 1810 \\
0 \cdot 1476\end{array}$ & $\begin{array}{r}39 \cdot 08 \\
4 \cdot 88\end{array}$ \\
\hline \multirow[t]{2}{*}{$\begin{array}{l}\text { BORIC ACID } \\
\text { PERIOD }\end{array}$} & & $\begin{array}{l}17 \mathrm{~V} \\
18, \\
19, \\
20, \\
21, \\
22,, \\
23,,\end{array}$ & $\begin{array}{l}0.50 \\
0.50 \\
0.50 \\
0.66 \\
0.66 \\
0.66 \\
1.00\end{array}$ & $\begin{array}{l}360 \\
315 \\
300 \\
425 \\
360 \\
410 \\
430\end{array}$ & $\begin{array}{c}\text { Amphoteric } \\
\text { ", } \\
, \\
\text { ” } \\
\text { ", }\end{array}$ & $\begin{array}{l}1 \cdot 0210 \\
1 \cdot 0258 \\
1 \cdot 0228 \\
1 \cdot 0205 \\
1 \cdot 0196 \\
1 \cdot 0205 \\
1 \cdot 0230\end{array}$ & $\begin{array}{l}1 \cdot 0123 \\
0 \cdot 9064 \\
0 \cdot 8436 \\
1 \cdot 1957 \\
1 \cdot 0123 \\
1 \cdot 0953 \\
1 \cdot 1489\end{array}$ & $\begin{array}{l}0.0561 \\
0.0491 \\
0.0468 \\
0.0663 \\
0.0561 \\
0.0558 \\
0.0584\end{array}$ & $\begin{array}{l}0 \cdot 1728 \\
0 \cdot 1512 \\
0 \cdot 1440 \\
0 \cdot 2040 \\
0 \cdot 1728 \\
0 \cdot 2091 \\
0 \cdot 2193\end{array}$ & $\begin{array}{l}4 \cdot 91 \\
5 \cdot 16 \\
4 \cdot 85 \\
5 \cdot 69 \\
4 \cdot 01 \\
6 \cdot 49 \\
6 \cdot 75\end{array}$ \\
\hline & $\begin{array}{l}\text { Total } \\
\text { Average }\end{array}$ & $\begin{array}{l}7 \text { days } \\
1 \text { day }\end{array}$ & $\begin{array}{l}4 \cdot 48 \\
0 \cdot 64\end{array}$ & $\begin{array}{r}2,600 \\
370\end{array}$ & & $1 \cdot 0218$ & $\begin{array}{l}7 \cdot 2145 \\
1 \cdot 0306\end{array}$ & $\begin{array}{l}0.3886 \\
0.0555\end{array}$ & $\begin{array}{l}1 \cdot 2732 \\
0 \cdot 1819\end{array}$ & $\begin{array}{r}37 \cdot 86 \\
5 \cdot 41\end{array}$ \\
\hline \multirow[t]{2}{*}{$\begin{array}{l}\text { BORAX } \\
\text { PERIOD }\end{array}$} & & $\begin{array}{l}24 \mathrm{v} \\
25, \\
26, \\
27, \\
28,\end{array}$ & $\begin{array}{l}1 \cdot 5 \\
1 \cdot 5 \\
1.5 \\
1.5 \\
1.5\end{array}$ & $\begin{array}{l}350 \\
320 \\
300 \\
450 \\
270\end{array}$ & $\begin{array}{l}\text { Acid } \\
\text {," } \\
, " \\
,\end{array}$ & $\begin{array}{l}1 \cdot 0228 \\
1 \cdot 0235 \\
1 \cdot 0225 \\
1 \cdot 0192 \\
1 \cdot 0263\end{array}$ & $\begin{array}{l}0.9240 \\
0 \cdot 8448 \\
0 \cdot 7920 \\
1 \cdot 1188 \\
0 \cdot 7128\end{array}$ & \begin{tabular}{|l|}
0.0574 \\
0.0529 \\
0.0492 \\
0.0738 \\
0.0443
\end{tabular} & $\begin{array}{l}0 \cdot 1733 \\
1 \cdot 1585 \\
0 \cdot 1485 \\
0 \cdot 2228 \\
0 \cdot 1337\end{array}$ & $\begin{array}{l}5 \cdot 17 \\
5 \cdot 00 \\
4 \cdot 52 \\
4 \cdot 12 \\
4 \cdot 31 \\
\end{array}$ \\
\hline & $\begin{array}{l}\text { Total } \\
\text { Average }\end{array}$ & $\begin{array}{l}5 \text { days } \\
1 \text { day }\end{array}$ & $\begin{array}{l}7 \cdot 5 \\
1 \cdot 5\end{array}$ & $\begin{array}{r}1,690 \\
338\end{array}$ & & $1 \cdot 0228$ & $\begin{array}{l}4 \cdot 3924 \\
0 \cdot 8785\end{array}$ & $\begin{array}{l}0 \cdot 2776 \\
0 \cdot 0555\end{array}$ & $\begin{array}{l}0.8367 \\
0.1673\end{array}$ & $\begin{array}{r}23 \cdot 12 \\
4 \cdot 62\end{array}$ \\
\hline \multirow[t]{2}{*}{$\begin{array}{r}\text { AFTER } \\
\text { PERIOD }\end{array}$} & & $\begin{array}{rl}29 & \mathrm{~V} \\
30 & , \\
31 & \dddot{\mathrm{V}} \\
1 & , \\
2 & ,\end{array}$ & & $\begin{array}{l}355 \\
335 \\
570 \\
435 \\
450\end{array}$ & $\begin{array}{c}\text { Acid } \\
\text {," } \\
, " \\
,\end{array}$ & $\begin{array}{l}1 \cdot 0219 \\
1 \cdot 0228 \\
1 \cdot 0188 \\
1 \cdot 0165 \\
1 \cdot 0205\end{array}$ & $\begin{array}{l}0 \cdot 8975 \\
0 \cdot 8469 \\
1 \cdot 4410 \\
1 \cdot 0996 \\
1 \cdot 1376\end{array}$ & \begin{tabular}{|l|}
0.0497 \\
0.0469 \\
0.0798 \\
0.0609 \\
0.0630
\end{tabular} & \begin{tabular}{|l|}
$0 \cdot 1438$ \\
$0 \cdot 1357$ \\
$0 \cdot 2308$ \\
$0 \cdot 1762$ \\
$0 \cdot 1823$
\end{tabular} & $\begin{array}{l}5 \cdot 01 \\
4 \cdot 95 \\
7 \cdot 10 \\
4 \cdot 86 \\
5 \cdot 55\end{array}$ \\
\hline & $\begin{array}{l}\text { Total } \\
\text { Average }\end{array}$ & $\begin{array}{l}5 \text { days } \\
1 \text { day }\end{array}$ & & $\begin{array}{r}2,145 \\
429\end{array}$ & & $1 \cdot 0201$ & $\begin{array}{l}5 \cdot 4226 \\
1 \cdot 0845\end{array}$ & $\begin{array}{l}0.3003 \\
0 \cdot 0600\end{array}$ & $\begin{array}{l}0 \cdot 8698 \\
0 \cdot 1737\end{array}$ & $\begin{array}{r}27 \cdot 47 \\
5 \cdot 49\end{array}$ \\
\hline
\end{tabular}




\section{TABLE II.}

upon the General Metabolism of Child A, aged $2 \frac{1}{2}$ years.

\begin{tabular}{|c|c|c|c|c|c|c|c|c|c|c|c|c|c|}
\hline \multicolumn{4}{|c|}{ FAECES } & \multirow[b]{2}{*}{$\begin{array}{l}\text { Nitrogen } \\
\text { of food }\end{array}$} & \multirow[b]{2}{*}{ Balance } & \multirow[b]{2}{*}{$\underset{\text { weight }}{\text { Body }}$} & \multicolumn{4}{|c|}{ PHOSPHORUS } & \multicolumn{3}{|c|}{ FAT } \\
\hline $\begin{array}{c}\text { Moist } \\
\mathrm{g} \\
\end{array}$ & $\begin{array}{c}\text { Dry } \\
\mathbf{g} \\
\end{array}$ & $\begin{array}{c}\text { Water } \\
\%\end{array}$ & $\begin{array}{c}\text { Nitro- } \\
\text { gen } \\
\text { g }\end{array}$ & & & & $\begin{array}{c}\text { Urine } \\
\mathbf{g} \\
\end{array}$ & $\begin{array}{c}\text { Faeces } \\
\mathbf{g} \\
\end{array}$ & $\begin{array}{c}\text { Food } \\
\mathrm{g} \\
\end{array}$ & $\begin{array}{c}\text { Balance } \\
\mathrm{g} \\
\end{array}$ & $\begin{array}{c}\text { Faeces } \\
\mathrm{g} \\
\end{array}$ & $\begin{array}{c}\text { Food } \\
\mathrm{g} \\
\end{array}$ & $\begin{array}{c}\text { Balance } \\
\mathbf{g} \\
\end{array}$ \\
\hline $\begin{array}{r}11 \\
88 \\
17 \\
114 \\
65 \\
60 \\
47 \\
116\end{array}$ & $\begin{array}{r}1 \cdot 9 \\
14 \cdot 5 \\
2 \cdot 7 \\
18 \cdot 0 \\
9 \cdot 9 \\
7 \cdot 6 \\
7 \cdot 7 \\
21 \cdot 4\end{array}$ & $\begin{array}{l}82 \cdot 7 \\
83 \cdot 5 \\
84 \cdot 1 \\
76 \cdot 0 \\
84 \cdot 8 \\
87 \cdot 3 \\
83 \cdot 8 \\
81 \cdot 4\end{array}$ & $\begin{array}{l}0 \cdot 11 \\
0 \cdot 86 \\
0 \cdot 16 \\
0 \cdot 06 \\
0.59 \\
0 \cdot 49 \\
0.50 \\
1.39\end{array}$ & $\begin{array}{l}7 \cdot 03 \\
7 \cdot 17 \\
7 \cdot 17 \\
7 \cdot 17 \\
7 \cdot 23 \\
7 \cdot 23 \\
6 \cdot 70 \\
6 \cdot 28\end{array}$ & $\begin{array}{l}+2.40 \\
+0.89 \\
+1.86 \\
+0.42 \\
+1.51 \\
+1.79 \\
+1.99 \\
+0.78\end{array}$ & $15 \cdot 28$ & $\begin{array}{l}0 \cdot 2765 \\
0 \cdot 3571 \\
0 \cdot 3974 \\
0 \cdot 5126 \\
0 \cdot 5069 \\
0 \cdot 3471 \\
0 \cdot 3044 \\
0 \cdot 2613\end{array}$ & $\begin{array}{l}0.0455 \\
0.3472 \\
0.0647 \\
0.4310 \\
0.2371 \\
0.1577 \\
0.1598 \\
0.4441\end{array}$ & $\begin{array}{l}0 \cdot 76 \\
0 \cdot 76 \\
0 \cdot 76 \\
0 \cdot 76 \\
0 \cdot 76 \\
0 \cdot 76 \\
0 \cdot 81 \\
0.80\end{array}$ & $\begin{array}{l}+0.44 \\
+0.06 \\
+0.30 \\
-0.18 \\
-0.02 \\
-0.26 \\
+0.35 \\
+0.09\end{array}$ & $\begin{array}{l}0 \cdot 42 \\
3 \cdot 23 \\
0 \cdot 60 \\
4 \cdot 01 \\
2 \cdot 21 \\
1 \cdot 85 \\
1 \cdot 87 \\
5 \cdot 19\end{array}$ & $\begin{array}{l}35 \cdot 57 \\
35 \cdot 57 \\
35 \cdot 57 \\
34 \cdot 96 \\
34 \cdot 96 \\
34 \cdot 96 \\
35 \cdot 16 \\
37 \cdot 46\end{array}$ & $\begin{array}{l}+35 \cdot 15 \\
+32 \cdot 34 \\
+34 \cdot 97 \\
+30 \cdot 95 \\
+32 \cdot 75 \\
+33 \cdot 11 \\
+33 \cdot 29 \\
+32 \cdot 27\end{array}$ \\
\hline $\begin{array}{r}518 \\
56\end{array}$ & $\begin{array}{l}83 \cdot 7 \\
10 \cdot 5\end{array}$ & $84 \cdot 0$ & $\begin{array}{l}5 \cdot 16 \\
0 \cdot 65\end{array}$ & $\begin{array}{r}55.98 \\
6.99\end{array}$ & $\begin{array}{r}+11 \cdot 64 \\
+1 \cdot 45\end{array}$ & $\begin{array}{c}-160 g . \\
L o s s \\
-23 g .\end{array}$ & $\begin{array}{l}2 \cdot 9633 \\
0 \cdot 3704\end{array}$ & $\begin{array}{l}1 \cdot 8871 \\
0 \cdot 2359\end{array}$ & $\begin{array}{l}6 \cdot 17 \\
0 \cdot 77\end{array}$ & $\begin{array}{l}+1 \cdot 34 \\
+0 \cdot 17\end{array}$ & $\begin{array}{r}19 \cdot 38 \\
2 \cdot 42\end{array}$ & $\begin{array}{r}284 \cdot 21 \\
35 \cdot 53\end{array}$ & $\begin{array}{r}+264 \cdot 83 \\
+33 \cdot 10\end{array}$ \\
\hline $\begin{array}{c}-\overline{83} \\
35 \\
95 \\
60 \\
109\end{array}$ & $\begin{array}{r}1 \overline{14} \cdot 9 \\
8 \cdot 4 \\
17 \cdot 9 \\
\overline{13 \cdot 4} \\
22 \cdot 2\end{array}$ & $\begin{array}{c}-\overline{82 \cdot 0} \\
76 \cdot 0 \\
81 \cdot 2 \\
- \\
7 \overline{7} \cdot 7 \\
79 \cdot 6\end{array}$ & $\begin{array}{l}0 \cdot \overline{8} \\
0 \cdot 47 \\
1 \cdot 01 \\
\overline{-} \\
0 \cdot 76 \\
1 \cdot 26\end{array}$ & $\begin{array}{l}6 \cdot 30 \\
6 \cdot 30 \\
6 \cdot 30 \\
6 \cdot 30 \\
6 \cdot 30 \\
6 \cdot 30 \\
6 \cdot 21\end{array}$ & $\begin{array}{l}+0.39 \\
+0.30 \\
-0.98 \\
+0.40 \\
-2.29 \\
-0.95 \\
-1.80\end{array}$ & $15 \cdot 12$ & $\begin{array}{l}0 \cdot 4784 \\
0 \cdot 3818 \\
0 \cdot 3636 \\
0 \cdot 5151 \\
0 \cdot 4784 \\
0 \cdot 4600 \\
0 \cdot 4833\end{array}$ & $\begin{array}{c}\overline{0} \overline{3144} \\
0 \cdot 1772 \\
0 \cdot 3777 \\
-\overline{-} \\
0 \cdot 2827 \\
0 \cdot 4684\end{array}$ & $\begin{array}{l}0 \cdot 80 \\
0 \cdot 80 \\
0 \cdot 80 \\
0 \cdot 8 \text { ? } \\
0 \cdot 80 \\
0 \cdot 80 \\
0 \cdot 81\end{array}$ & $\begin{array}{l}+0.32 \\
+0.10 \\
+0.26 \\
+0.09 \\
+0.32 \\
+0.06 \\
-0.14\end{array}$ & $\begin{array}{l}- \\
3 \cdot 60 \\
2 \cdot 03 \\
4 \cdot 32 \\
-- \\
3 \cdot 24 \\
5 \cdot 37\end{array}$ & $\begin{array}{l}37 \cdot 46 \\
37 \cdot 46 \\
37 \cdot 46 \\
37 \cdot 46 \\
37 \cdot 46 \\
37 \cdot 46 \\
35 \cdot 37\end{array}$ & $\begin{array}{l}+37 \cdot 46 \\
+33 \cdot 86 \\
+35 \cdot 43 \\
+33 \cdot 14 \\
+37 \cdot 46 \\
+34 \cdot 22 \\
+30 \cdot 00\end{array}$ \\
\hline $\begin{array}{r}382 \\
54\end{array}$ & $\begin{array}{l}76 \cdot 8 \\
10 \cdot 9\end{array}$ & $80 \cdot 0$ & $\begin{array}{l}4 \cdot 34 \\
0 \cdot 62\end{array}$ & $\begin{array}{r}44 \cdot 01 \\
6 \cdot 29\end{array}$ & $\begin{array}{r}1.81 \\
+0.26\end{array}$ & $\begin{array}{c}+300 \mathrm{~g} . \\
\text { Gain } \\
+43 g .\end{array}$ & $\begin{array}{l}3 \cdot 1606 \\
0 \cdot 4515\end{array}$ & $\begin{array}{l}1 \cdot 6204 \\
0 \cdot 2315\end{array}$ & $\begin{array}{l}5 \cdot 61 \\
0 \cdot 80\end{array}$ & $\begin{array}{l}+0.83 \\
+0 \cdot 12\end{array}$ & $\begin{array}{r}18 \cdot 56 \\
2 \cdot 65\end{array}$ & $\begin{array}{r}260 \cdot 13 \\
37 \cdot 16\end{array}$ & $\begin{array}{r}+241 \cdot 57 \\
+34 \cdot 51\end{array}$ \\
\hline $\begin{array}{l}10 \\
27 \\
74 \\
61 \\
72\end{array}$ & $\begin{array}{r}1 \cdot 5 \\
5 \cdot 2 \\
12 \cdot 5 \\
11 \cdot 3 \\
15 \cdot 0\end{array}$ & $\begin{array}{l}85 \cdot 0 \\
80 \cdot 7 \\
83 \cdot 1 \\
80 \cdot 0 \\
79 \cdot 2\end{array}$ & $\begin{array}{l}0 \cdot 10 \\
0 \cdot 34 \\
0 \cdot 81 \\
0 \cdot 73 \\
0 \cdot 97\end{array}$ & $\begin{array}{l}6 \cdot 21 \\
6 \cdot 25 \\
6 \cdot 41 \\
6 \cdot 41 \\
5 \cdot 71\end{array}$ & $\begin{array}{l}+0.94 \\
+0.91 \\
+1.08 \\
+1.56 \\
+0.43\end{array}$ & $15 \cdot 42$ & $\begin{array}{l}0 \cdot 4424 \\
0 \cdot 4045 \\
0 \cdot 3792 \\
0 \cdot 5688 \\
0 \cdot 3413\end{array}$ & $\begin{array}{l}0 \cdot 0338 \\
0 \cdot 1172 \\
0 \cdot 2816 \\
0 \cdot 2546 \\
0 \cdot 3370\end{array}$ & $\begin{array}{l}0.81 \\
0.81 \\
0.81 \\
0.81 \\
0.81\end{array}$ & $\begin{array}{l}+0.33 \\
+0.29 \\
+0.15 \\
-0.01 \\
+0.13\end{array}$ & $\begin{array}{l}0 \cdot 31 \\
1 \cdot 08 \\
2 \cdot 59 \\
2 \cdot 35 \\
3 \cdot 12\end{array}$ & $\begin{array}{l}35 \cdot 17 \\
35 \cdot 17 \\
41 \cdot 42 \\
41 \cdot 42 \\
41 \cdot 42\end{array}$ & $\begin{array}{l}+35 \cdot 06 \\
+34 \cdot 29 \\
+38 \cdot 83 \\
+39 \cdot 07 \\
+38 \cdot 30\end{array}$ \\
\hline $\begin{array}{r}244 \\
49\end{array}$ & $\begin{array}{r}45 \cdot 5 \\
9 \cdot 1\end{array}$ & $81 \cdot 4$ & $\begin{array}{l}2.95 \\
0.59\end{array}$ & $\begin{array}{r}30 \cdot 99 \\
6 \cdot 20\end{array}$ & $\begin{array}{l}+4.92 \\
+0.98\end{array}$ & $\begin{array}{c}+30 g . \\
\text { Gain } \\
+6 g .\end{array}$ & $\begin{array}{l}2 \cdot 1362 \\
0 \cdot 4272\end{array}$ & $\begin{array}{l}1 \cdot 0242 \\
0 \cdot 2048\end{array}$ & $\begin{array}{l}4.05 \\
0.81\end{array}$ & $\begin{array}{l}+0.89 \\
+0.18\end{array}$ & $\begin{array}{l}9 \cdot 45 \\
1 \cdot 89\end{array}$ & $\begin{array}{r}195 \cdot 00 \\
39 \cdot 00\end{array}$ & $\begin{array}{r}+185 \cdot 55 \\
+37 \cdot 11\end{array}$ \\
\hline \begin{tabular}{|}
$\overline{48}$ \\
80 \\
$\overline{131}$
\end{tabular} & $\begin{array}{l}\overline{11 \cdot 9} \\
16 \cdot 4 \\
-\overline{27 \cdot 0}\end{array}$ & $\begin{array}{l}\overline{75 \cdot 2} \\
79 \cdot 5 \\
\overline{79 \cdot 4}\end{array}$ & $\begin{array}{l}0 \overline{78} \\
1 \cdot 07 \\
\overline{1 \cdot 77}\end{array}$ & $\begin{array}{l}5 \cdot 74 \\
6 \cdot 43 \\
6 \cdot 44 \\
6 \cdot 44 \\
6 \cdot 44\end{array}$ & $\begin{array}{l}+0.73 \\
+0.70 \\
-1.73 \\
+1.58 \\
-0.88\end{array}$ & $15 \cdot 45$ & $\begin{array}{l}0 \cdot 4075 \\
0 \cdot 3846 \\
0 \cdot 6544 \\
0 \cdot 4994 \\
0 \cdot 5766\end{array}$ & $\begin{array}{c}\overline{0} \cdot \overline{2812} \\
0 \cdot 3875 \\
\overline{0}-\overline{6380}\end{array}$ & $\begin{array}{l}0 \cdot 81 \\
0 \cdot 81 \\
0 \cdot 80 \\
0.80 \\
0.80\end{array}$ & $\begin{array}{l}+0.40 \\
+0.14 \\
-0.24 \\
+0.30 \\
-0.41\end{array}$ & $\begin{array}{l}- \\
2 \cdot 31 \\
3 \cdot 18 \\
- \\
5 \cdot 24\end{array}$ & $\begin{array}{l}41 \cdot 68 \\
41 \cdot 68 \\
41 \cdot 68 \\
41 \cdot 68 \\
41 \cdot 68\end{array}$ & $\begin{array}{l}+41 \cdot 68 \\
+39.37 \\
+38.50 \\
+41.68 \\
+36.44\end{array}$ \\
\hline $\begin{array}{r}259 \\
52\end{array}$ & $\begin{array}{l}55 \cdot 3 \\
11 \cdot 0\end{array}$ & $78 \cdot 6$ & $\begin{array}{l}3 \cdot 62 \\
0 \cdot 72\end{array}$ & $\begin{array}{r}31 \cdot 49 \\
6 \cdot 29\end{array}$ & $\begin{array}{r}+0.40 \\
0.08\end{array}$ & \pm 0 & $\begin{array}{l}2.5225 \\
0.5045\end{array}$ & $\begin{array}{l}1 \cdot 3067 \\
0 \cdot 2613\end{array}$ & $\begin{array}{l}4 \cdot 02 \\
0.80\end{array}$ & $\begin{array}{l}+0.19 \\
+0.04\end{array}$ & $\begin{array}{r}10 \cdot 73 \\
2 \cdot 14\end{array}$ & $\begin{array}{r}208 \cdot 40 \\
41 \cdot 68\end{array}$ & $\begin{array}{r}+197 \cdot 67 \\
+39.53\end{array}$ \\
\hline
\end{tabular}


The results expressed in the above table are graphically represented in the following curves:

\section{Curve I.,}

showing the influence of boric acid and borax upon nitrogen and phosphorus metabolism, etc.

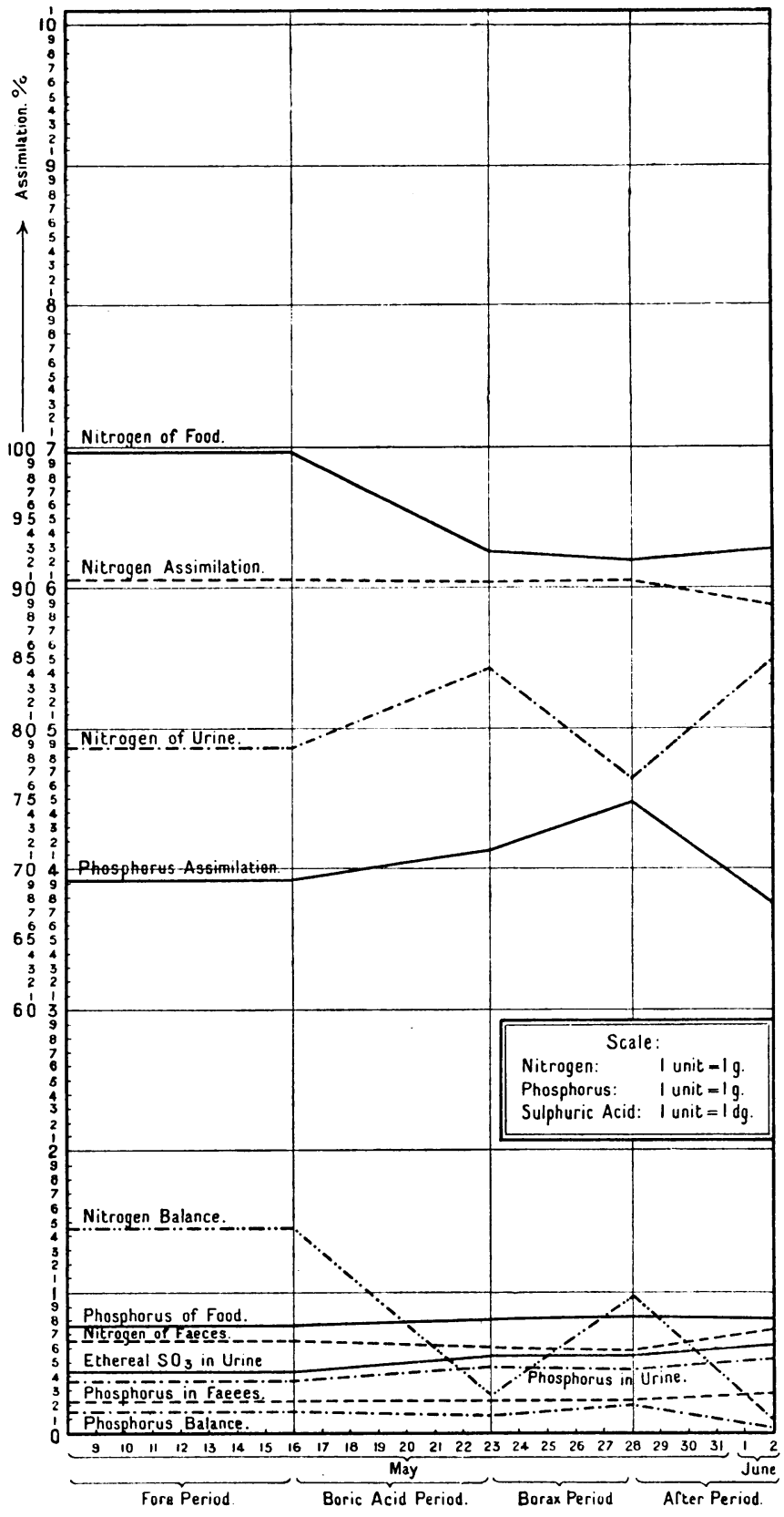


Curve II.,

showing the influence of boric acid and borax upon fat-assimilation and the quantity of faeces and urine.

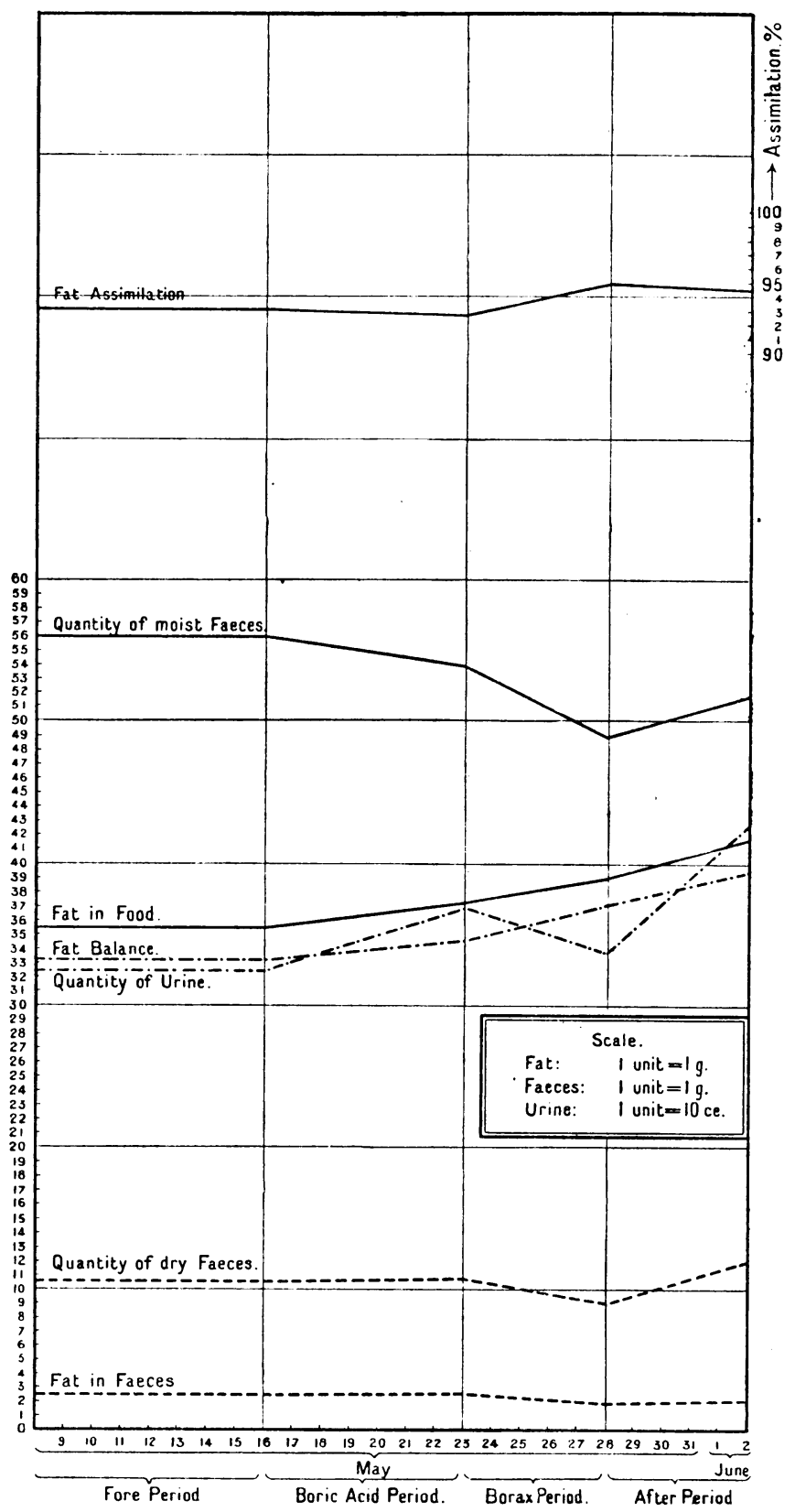




\section{Observation I. Child A.}

The child was a healthy boy aged $2 \frac{1}{2}$ years, weighed $15 \cdot 3$ kilos, and remained in good health throughout the whole observation. He consumed daily as follows, $200 \mathrm{~g}$. of bread, 550 c.c. of milk, 20 g. of butter, 30 g. of meat, 50 g. of apple compote, $10 \mathrm{~g}$. of sugar, $50 \mathrm{ccm}$. of water, $5 \mathrm{~g}$. of toffee. This diet was very well taken and adhered to throughout the experiment. The whole observation extended over twenty-five days, eight days being taken as a fore period, and five days as an after period. The intermediate period of twelve days consisted of a boric acid period of seven days and a borax period of five days. The pure substances, boric acid or borax as the case may be, were added to 500 c.c. of the daily milk early in the morning and were administered as shown in the following table:

Boric Acid Period.

3 days : 0.5 g. per diem $=1$ in 1000 in Milk $=1$ in 1800 in total Food and Drink.

3 days : $0.66 \mathrm{~g}$. " $=1$ in $760 \quad,=1$ in $1370 \quad, \quad "$,

1 day: 1 g. $\quad, \quad=1$ in $500 \quad,=1$ in $900 \quad " \quad, \quad "$

Borax Period.

5 days : 1.5 g. per diem $=1$ in 330 in Milk $=1$ in 600 in total Food and Drink.

It may be noted here that the maximum medicinal dose for this child would be $0.2 \mathrm{~g}$. of boric acid and $0.27 \mathrm{~g}$. of borax, also that the quantities given are greatly in excess of those required for the preservation of milk ${ }^{1}$.

The analytical results obtained throughout the observation are recorded in Table II. pp. 176-7.

Referring to the tables and curves relating to child A, we purpose classifying our remarks under the following headings :

\section{Nitrogen Metabolism.}

In the fore period the daily quantity of nitrogen taken in the food was $6.99 \mathrm{~g}$., of which $0.65 \mathrm{~g}$. were not assimilated, being lost with the faeces, corresponding to $9.3 \%$. The assimilation of nitrogen in the fore period amounted therefore to $9070 \%$.

With the urine $4.88 \mathrm{~g}$. of nitrogen were excreted, and if this amount be subtracted from the amount assimilated we obtain a daily balance of +1.45 g. nitrogen.

1 Vide Droop Richmond and Harrison, Analyst, 1900, p. 116. 
To avoid repetition we give the results with regard to the nitrogen balance and assimilation during the different periods in tabular form:

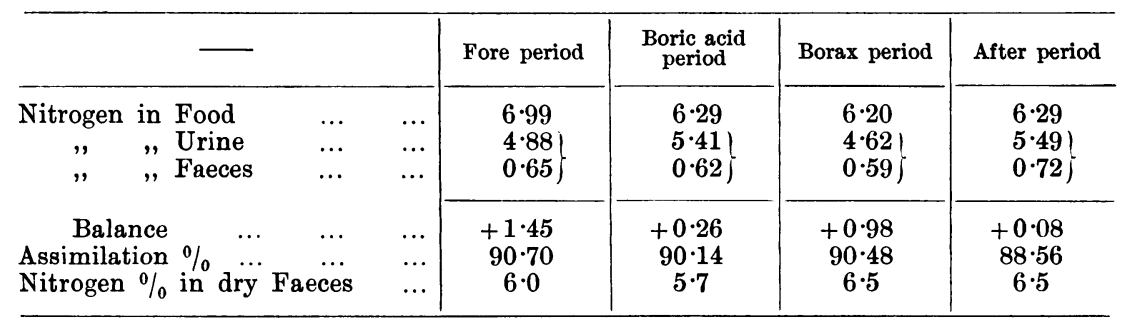

From these results we are justified in concluding that neither boric acid nor borax exerts any influence upon the assimilation of proteids. The tendency of the body to reach nitrogenous equilibrium is clearly shown in the balance figures. From the amount of nitrogen excreted in the urine during the respective periods we may perhaps draw the conclusion that boric acid in this instance tends slightly to increase and borax slightly to inhibit proteid katabolism.

\section{Phosphorus Metabolism.}

The daily average quantity of phosphorus in the food during the fore period was $0.77 \mathrm{~g}$., of which $0 \cdot 2359 \mathrm{~g}$. were lost, being excreted with the faeces. Phosphorus was therefore assimilated ${ }^{1}$ to the extent of $69.36 \%$. The relative excretion etc., of phosphorus in the four periods we give in tabular form.

\begin{tabular}{|c|c|c|c|c|c|c|c|}
\hline & & & & Fore period & $\begin{array}{c}\text { Boric acid } \\
\text { period }\end{array}$ & Borax period & After period \\
\hline $\begin{array}{c}\text { Phosphorus in } \\
, "\end{array}$ & $\begin{array}{l}\text { n Food } \\
\text { Urine } \\
\text {, Faeces }\end{array}$ & $\begin{array}{l}\cdots \\
\cdots \\
\cdots\end{array}$ & $\begin{array}{l}\cdots \\
\cdots \\
\cdots\end{array}$ & $\left.\begin{array}{l}0 \cdot 77 \\
0 \cdot 3704 \\
0 \cdot 2359\end{array}\right\}$ & $\left.\begin{array}{l}0 \cdot 80 \\
0 \cdot 4515 \\
0 \cdot 2315\end{array}\right\}$ & $\left.\begin{array}{l}0.81 \\
0.4272 \\
0.2048\end{array}\right\}$ & $\left.\begin{array}{l}0 \cdot 80 \\
0 \cdot 5045 \\
0 \cdot 2613\end{array}\right\}$ \\
\hline $\begin{array}{l}\text { Balance } \\
\text { Assimilation } \\
\text { Phosphorus } 0\end{array}$ & $\begin{array}{l}\cdots \\
\% \\
\%\end{array}$ & $\begin{array}{l}\ldots \\
\ldots \\
\text { Faeces }\end{array}$ & $\begin{array}{l}\cdots \\
\cdots \\
\cdots\end{array}$ & $\begin{array}{c}+0 \cdot 17 \\
69 \cdot 36 \\
2 \cdot 2\end{array}$ & $\begin{array}{c}+0 \cdot 12 \\
71 \cdot 06 \\
2 \cdot 1\end{array}$ & $\begin{array}{c}+0 \cdot 18 \\
74 \cdot 82 \\
2 \cdot 2\end{array}$ & $\begin{array}{c}+0.04 \\
67.34 \\
2.4\end{array}$ \\
\hline
\end{tabular}

These figures show that the phosphorus metabolism was not affected by boric acid and borax. The assimilation of phosphorus was if anything improved during the drug periods.

1 The term assimilation in this sense is perhaps not strictly correct, we do not purpose however entering here into the actual source of the faecal phosphorus. 


\section{Fat Assimilation.}

The daily quantity of fat in the food during the fore period was $35.53 \mathrm{~g}$. The fat excreted with the faeces was 2.42 grammes. The assimilation therefore amounted to $93 \cdot 19 \%$. These results and those of the following periods are recorded in tabular form as follows :

\begin{tabular}{|c|c|c|c|c|c|c|}
\hline & & & Fore period & $\begin{array}{c}\text { Boric acid } \\
\text { period }\end{array}$ & Borax period & After period \\
\hline $\begin{array}{cc}\text { Fat in Food } & \ldots \\
,, & \text { Faeces }\end{array}$ & $\begin{array}{l}\ldots \\
\cdots\end{array}$ & $\ldots$ & $\begin{array}{r}35 \cdot 53 \\
2 \cdot 42\end{array}$ & $\begin{array}{r}37 \cdot 16 \\
2 \cdot 65\end{array}$ & $\begin{array}{r}39 \cdot 00 \\
1 \cdot 89\end{array}$ & $\begin{array}{r}41 \cdot 68 \\
2 \cdot 14\end{array}$ \\
\hline 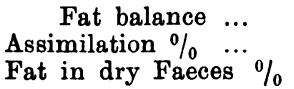 & $\begin{array}{l}\cdots \\
\cdots \\
\cdots\end{array}$ & $\begin{array}{l}. . \\
\cdots \\
\cdots\end{array}$ & $\begin{array}{c}+33 \cdot 10 \\
93 \cdot 19 \\
23 \cdot 0\end{array}$ & $\begin{array}{c}+34 \cdot 51 \\
92 \cdot 87 \\
24 \cdot 3\end{array}$ & $\begin{array}{c}+37 \cdot 11 \\
95 \cdot 19 \\
20 \cdot 8\end{array}$ & $\begin{array}{c}+39 \cdot 53 \\
94 \cdot 87 \\
19 \cdot 5\end{array}$ \\
\hline
\end{tabular}

It will be seen from these figures that the amount of fat retained by the body rose with the amount of fat in the food. If anything the assimilation of fat was increased during the borax period.

The chief remaining points of interest brought out by this observation are as follows ${ }^{1}$ :

The quantity of urine underwent slight variations during the drug periods in the direction of an increase. The increase was more marked during the boric acid period.

The specific gravity diminished as the volume increased.

The reaction alternating between acid and amphoteric (litmus) during the fore period remained constantly amphoteric during the boric acid period and constantly acid during the borax period.

The quantity of faeces remained practically unaltered with the exception of the borax period, in which the average daily quantity is slightly decreased.

The increase in uric acid is too slight to permit of any conclusions being drawn from it.

The quantity of total sulphuric acid increased slightly in the boric acid period, indicating with the slight increase of nitrogen in the urine a tendency to stimulate proteid katabolism.

The ethereal sulphates were slightly increased during both periods to an equal extent. Intestinal putrefaction was therefore certainly not diminished by either substance, as was also shown by the comparative indoxyl-reactions.

1 In these remarks throughout the entire paper we refer to the average daily excretion in question. 
The boric acid could easily be demonstrated in the urine on the first day of its administration and disappeared completely in the course of the second day of the after period. These results show clearly that both boric acid and borax are rapidly eliminated from the body, and confirm the results of previous workers 1 .

During the boric acid and borax periods the child gained in weight.

The results relevant to the observations made above are summarised in the following table:-

Table II A.

\begin{tabular}{|c|c|c|c|c|c|c|c|c|}
\hline & $\mid$\begin{tabular}{c|} 
Nitrogen \\
assimilation, \\
$\%$
\end{tabular} & $\begin{array}{l}\% 0 \mathrm{~N} . \\
\text { of dry } \\
\text { faeces }\end{array}$ & \begin{tabular}{|} 
Phosphorus \\
assimilation, \\
$\% \%$
\end{tabular} & $\begin{array}{l}\% \mathbf{P} . \\
\text { of dry } \\
\text { faeces }\end{array}$ & $\underset{0 \%}{\text { assimilation, }}$ & $\begin{array}{l}\% \text { Fat } \\
\text { of dry } \\
\text { faeces }\end{array}$ & $\frac{\mathbf{A}^{*}}{\overline{\mathbf{B}}}$ & $\frac{\mathrm{N}+}{\mathrm{SO}_{3}}$ \\
\hline $\begin{array}{l}\text { Fore period } \\
\text { Boric acid period } \\
\text { Borax period } \ldots \\
\text { After period } \ldots\end{array}$ & $\begin{array}{l}90 \cdot 70 \\
90 \cdot 14 \\
90 \cdot 48 \\
88 \cdot 56\end{array}$ & $\begin{array}{l}6 \cdot 0 \\
5 \cdot 7 \\
6 \cdot 5 \\
6 \cdot 5\end{array}$ & $\begin{array}{l}69 \cdot 36 \\
71 \cdot 06 \\
74 \cdot 82 \\
67 \cdot 34\end{array}$ & $\begin{array}{l}2 \cdot 2 \\
2 \cdot 1 \\
2 \cdot 2 \\
2 \cdot 4\end{array}$ & $\begin{array}{l}93 \cdot 19 \\
92 \cdot 87 \\
95 \cdot 19 \\
94 \cdot 87\end{array}$ & $\begin{array}{l}23 \cdot 0 \\
24 \cdot 3 \\
20 \cdot 8 \\
19 \cdot 5\end{array}$ & $\begin{array}{l}20 \cdot 2 \\
17 \cdot 6 \\
14 \cdot 4 \\
17 \cdot 1\end{array}$ & $\begin{array}{l}5 \cdot 2 \\
5 \cdot 2 \\
5 \cdot 3 \\
5 \cdot 1\end{array}$ \\
\hline
\end{tabular}

$$
\begin{aligned}
& * \frac{\mathrm{A}}{\mathrm{B}}=\frac{\text { Inorganic } \mathrm{SO}_{3}}{\text { Ethereal } \mathrm{SO}_{3}} \\
& +\frac{\mathrm{N}}{\mathrm{SO}_{3}}=\frac{\text { Nitrogen of Urine }}{\mathrm{SO}_{3} \text { of Urine }} .
\end{aligned}
$$

\section{Observation II. Child B.}

The child was a healthy boy aged 5 years, weighing 18.5 kilos, and remained in good health during the whole observation. He consumed daily $250 \mathrm{~g}$. of bread, 600 c.c. of milk, 20 g. of butter, 50 g. of meat, 50 g. of apple compote, 10 g. of sugar, 50 c.c. of water, and $5 \mathrm{~g}$. of toffee. The whole observation lasted for twenty-two days. The fore period in this case lasted for 5 days, otherwise the arrangement and quantity of boric acid and borax given were the same as in Observation I. These substances were administered as shown in the following table :

Boric Acid Period.

3 days : 0.5 g. per diem $=1$ in 1000 Milk, 1 in 2000 total Food and Drink.

3 days : $0.66 \mathrm{~g} . \quad,=1$ in $760 \quad, 1$ in $1500, ", \quad$,

1 day : $1.0 \mathrm{~g} . \quad, \quad=1$ in $500 \quad, 1$ in $1000, "$,

Borax Period.

5 days : $1.5 \mathrm{~g} . \quad, \quad=1$ in $330, \quad 1$ in $660, "$,

It may be noted here that the maximal medicinal dose for this child is in the case of boric acid $0.29 \mathrm{~g}$., of borax $0.38 \mathrm{~g}$., and that the quantities given, as in the last observation, are greatly in excess of those which would be required as a food preservative.

The analytical results obtained throughout this observation are recorded in the following table :-

1 Chittenden and Gies (loc. cit.), where also references to former observers are given. 
184 Influence of Boric Acid and Borax, etc.

\section{TABLE III.}

Showing the Influence of Boric Acid AND

\begin{tabular}{|c|c|c|c|c|c|c|c|c|c|c|}
\hline \multirow[b]{2}{*}{ PERIOD } & & \multirow[b]{2}{*}{ Date } & \multirow{3}{*}{$\begin{array}{r}\text { Dose } \\
\mathrm{g} \\
\end{array}$} & \multicolumn{7}{|c|}{ URINE } \\
\hline & & & & $\begin{array}{c}\text { Quantity } \\
\text { c.c. }\end{array}$ & Reaction & $\begin{array}{l}\text { Specific } \\
\text { gravity }\end{array}$ & $\begin{array}{c}\text { Total } \\
\text { sulphuric } \\
\text { acid } \\
\mathbf{g}\end{array}$ & $\begin{array}{c}\text { Ethereal } \\
\text { sulphuric } \\
\text { acid } \\
\mathbf{g}\end{array}$ & $\begin{array}{l}\text { Uric } \\
\text { acid } \\
\mathrm{g}\end{array}$ & $\begin{array}{c}\text { Nitrogen } \\
\mathrm{g} \\
\end{array}$ \\
\hline \multirow[t]{2}{*}{$\begin{array}{c}\text { FORE } \\
\text { PERIOD }\end{array}$} & & $\begin{array}{ll}12 & \mathrm{~V} . \\
13 & , \\
14 & , \\
15 & , \\
16 & ,\end{array}$ & & $\begin{array}{l}510 \\
395 \\
390 \\
450 \\
470\end{array}$ & $\begin{array}{c}\text { Acid } \\
\text { Amphoteric } \\
,, \\
,,\end{array}$ & $\begin{array}{l}1 \cdot 0194 \\
1 \cdot 0240 \\
1 \cdot 0185 \\
1 \cdot 0228 \\
1 \cdot 0226\end{array}$ & $\begin{array}{l}1 \cdot 4412 \\
1 \cdot 1762 \\
1 \cdot 1020 \\
1 \cdot 2715 \\
1 \cdot 3281\end{array}$ & $\begin{array}{l}0 \cdot 0899 \\
0 \cdot 0696 \\
0 \cdot 0687 \\
0 \cdot 0793 \\
0 \cdot 0821\end{array}$ & $\begin{array}{l}0 \cdot 1035 \\
0 \cdot 0802 \\
0 \cdot 0792 \\
0 \cdot 0914 \\
0 \cdot 0954\end{array}$ & $\begin{array}{l}6 \cdot 47 \\
6 \cdot 59 \\
4 \cdot 64 \\
7 \cdot 21 \\
7 \cdot 41\end{array}$ \\
\hline & $\begin{array}{l}\text { Total } \\
\text { Average }\end{array}$ & $\begin{array}{l}5 \text { days } \\
1 \text { day }\end{array}$ & & $\begin{array}{r}2,215 \\
440\end{array}$ & & $1 \cdot 0214$ & $\begin{array}{l}6 \cdot 3190 \\
1 \cdot 2638\end{array}$ & $\begin{array}{l}0 \cdot 3896 \\
0 \cdot 0779\end{array}$ & $\begin{array}{l}0.4497 \\
0.0899\end{array}$ & $\begin{array}{r}32 \cdot 32 \\
6 \cdot 46\end{array}$ \\
\hline \multirow[t]{2}{*}{$\begin{array}{l}\text { BORIC ACID } \\
\text { PERIOD }\end{array}$} & & $\begin{array}{ll}17 & \mathrm{~V} . \\
18 & , \\
19 & , \\
20 & , \\
21 & , \\
22 & , \\
23 & ,\end{array}$ & $\begin{array}{l}0.50 \\
0.50 \\
0.50 \\
0.66 \\
0.66 \\
0.66 \\
1.00\end{array}$ & $\begin{array}{l}365 \\
400 \\
450 \\
305 \\
240 \\
600 \\
430\end{array}$ & $\begin{array}{l}\text { Acid } \\
\text {,, } \\
\text {,, } \\
\text {,, } \\
\text {,, }\end{array}$ & $\begin{array}{l}1 \cdot 0275 \\
1 \cdot 0266 \\
1 \cdot 0225 \\
1 \cdot 0254 \\
1 \cdot 0175 \\
1 \cdot 0244 \\
1 \cdot 0280\end{array}$ & $\begin{array}{l}1 \cdot 1100 \\
1 \cdot 2165 \\
1 \cdot 3685 \\
0 \cdot 9276 \\
0 \cdot 7299 \\
1 \cdot 9629 \\
1 \cdot 4174\end{array}$ & $\begin{array}{l}0 \cdot 0744 \\
0 \cdot 0816 \\
0 \cdot 0918 \\
0 \cdot 0622 \\
0 \cdot 0489 \\
0 \cdot 1092 \\
0 \cdot 0783\end{array}$ & $\begin{array}{l}0 \cdot 0631 \\
0 \cdot 0692 \\
0 \cdot 0779 \\
0 \cdot 0528 \\
0 \cdot 0415 \\
0 \cdot 2250 \\
0 \cdot 1613\end{array}$ & $\begin{array}{r}6 \cdot 66 \\
6 \cdot 94 \\
6 \cdot 84 \\
5 \cdot 26 \\
3 \cdot 18 \\
10 \cdot 27 \\
7 \cdot 16\end{array}$ \\
\hline & $\begin{array}{l}\text { Total } \\
\text { Average }\end{array}$ & $\begin{array}{l}7 \text { days } \\
1 d a y\end{array}$ & $\begin{array}{l}4 \cdot 48 \\
0 \cdot 64\end{array}$ & $\begin{array}{r}2,790 \\
398\end{array}$ & & $1 \cdot 0245$ & $\begin{array}{l}8 \cdot 7328 \\
1 \cdot 2475\end{array}$ & $\begin{array}{l}0 \cdot 5464 \\
0 \cdot 0780\end{array}$ & $\begin{array}{l}0.6908 \\
0 \cdot 0987\end{array}$ & $\begin{array}{r}46 \cdot 31 \\
6 \cdot 61\end{array}$ \\
\hline \multirow[t]{2}{*}{$\begin{array}{c}\text { BORAX } \\
\text { PERIOD }\end{array}$} & & $\begin{array}{ll}24 & \mathrm{~V} . \\
25 & , \\
26 & , \\
27 & , \\
28 & ,\end{array}$ & $\begin{array}{l}1.5 \\
1.5 \\
1.5 \\
1.5 \\
1.5\end{array}$ & $\begin{array}{l}320 \\
410 \\
365 \\
430 \\
300\end{array}$ & $\begin{array}{c}\text { Acid } \\
\text {,, } \\
,, \\
,, \\
\text {, }\end{array}$ & $\begin{array}{l}1 \cdot 0228 \\
1 \cdot 0248 \\
1 \cdot 0255 \\
1 \cdot 0236 \\
1 \cdot 0290\end{array}$ & $\begin{array}{l}1 \cdot 0496 \\
1 \cdot 3448 \\
1 \cdot 1972 \\
1 \cdot 4104 \\
0 \cdot 9840\end{array}$ & $\begin{array}{l}0.0717 \\
0.0918 \\
0.0818 \\
0.0963 \\
0.0672\end{array}$ & $\begin{array}{l}0.0720 \\
0.0923 \\
0.0821 \\
0.0968 \\
0.0675\end{array}$ & $\begin{array}{l}6 \cdot 22 \\
7 \cdot 23 \\
6 \cdot 04 \\
6 \cdot 87 \\
5 \cdot 38\end{array}$ \\
\hline & $\begin{array}{l}\text { Total } \\
\text { Average }\end{array}$ & $\begin{array}{l}5 \text { days } \\
1 \text { day }\end{array}$ & $\begin{array}{l}7 \cdot 5 \\
1 \cdot 5\end{array}$ & $\begin{array}{r}1,825 \\
365\end{array}$ & & $1 \cdot 0251$ & $\begin{array}{l}5 \cdot 9860 \\
1 \cdot 1972\end{array}$ & $\begin{array}{c}0 \cdot 4088 \\
0 \cdot 0817\end{array}$ & $\begin{array}{l}0.4107 \\
0 \cdot 0821\end{array}$ & $\begin{array}{r}31 \cdot 74 \\
6 \cdot 35\end{array}$ \\
\hline \multirow[t]{2}{*}{$\begin{array}{c}\text { AETER } \\
\text { PERIOD }\end{array}$} & & $\begin{array}{rr}29 & \mathrm{~V} . \\
30 & , \\
31 & , \\
1 & , \\
2 & , \\
\end{array}$ & & $\begin{array}{l}395 \\
335 \\
300 \\
425 \\
405 \\
\end{array}$ & $\begin{array}{c}\text { Acid } \\
,, \\
,, \\
,, \\
\end{array}$ & $\begin{array}{l}1 \cdot 0195 \\
1 \cdot 0282 \\
1 \cdot 0300 \\
1 \cdot 0232 \\
1 \cdot 0215\end{array}$ & $\begin{array}{l}1 \cdot 2403 \\
1 \cdot 0519 \\
0 \cdot 9420 \\
1 \cdot 3345 \\
1 \cdot 2717\end{array}$ & $\begin{array}{l}0.0672 \\
0.0569 \\
0.0510 \\
0.0722 \\
0.0688\end{array}$ & $\begin{array}{l}0 \cdot 1185 \\
0 \cdot 1005 \\
0 \cdot 0900 \\
0 \cdot 1275 \\
0 \cdot 1215\end{array}$ & $\begin{array}{l}5 \cdot 21 \\
6 \cdot 41 \\
6 \cdot 79 \\
7 \cdot 08 \\
6 \cdot 49\end{array}$ \\
\hline & $\begin{array}{l}\text { Total } \\
\text { Average }\end{array}$ & $\begin{array}{l}5 \text { days } \\
1 \text { day }\end{array}$ & & $\begin{array}{r}1,860 \\
372\end{array}$ & & $1 \cdot 0245$ & $\begin{array}{l}5 \cdot 8404 \\
1 \cdot 1681\end{array}$ & $\begin{array}{l}0.3161 \\
0.0632\end{array}$ & $\begin{array}{l}0.5580 \\
0 \cdot 1116\end{array}$ & $\begin{array}{r}31 \cdot 98 \\
6 \cdot 39\end{array}$ \\
\hline
\end{tabular}




\section{TABLE III.}

Borax upon the General Metabolism of Child $B$.

\begin{tabular}{|c|c|c|c|c|c|c|c|c|c|c|c|c|c|}
\hline \multicolumn{4}{|c|}{ FAECES } & \multirow[b]{2}{*}{$\begin{array}{c}\text { Nitro- } \\
\text { gen of } \\
\text { food }\end{array}$} & \multirow[b]{2}{*}{$\begin{array}{c}\text { Balance } \\
\mathrm{g} \\
\end{array}$} & \multirow[b]{2}{*}{$\begin{array}{r}\begin{array}{c}\text { Body } \\
\text { weight }\end{array} \\
\text { kg } \\
\end{array}$} & \multicolumn{4}{|c|}{ PHOSPHORUS } & \multicolumn{3}{|c|}{ FAT } \\
\hline $\begin{array}{c}\text { Moist } \\
\mathrm{g} \\
\end{array}$ & $\begin{array}{c}\text { Dry } \\
\mathrm{g}\end{array}$ & $\underset{\%}{\text { Water }}$ & $\begin{array}{l}\text { Nitro- } \\
\text { gen } \\
\text { g }\end{array}$ & & & & $\begin{array}{c}\text { Urine } \\
\end{array}$ & $\begin{array}{c}\text { Faeces } \\
\mathbf{g} \\
\end{array}$ & $\begin{array}{c}\text { Food } \\
\mathrm{g} \\
\end{array}$ & $\begin{array}{c}\text { Balance } \\
\mathrm{g} \\
\end{array}$ & $\begin{array}{c}\text { Faeces } \\
\mathrm{g} \\
\end{array}$ & $\begin{array}{c}\text { Food } \\
\mathrm{g} \\
\end{array}$ & $\begin{array}{c}\text { Balance } \\
\mathbf{g} \\
\end{array}$ \\
\hline $\begin{array}{r}\overline{110} \\
\overline{94} \\
107\end{array}$ & $\begin{array}{l}\overline{-} \\
27 \cdot 2 \\
2 \overline{-} \cdot 2 \\
25 \cdot 2\end{array}$ & $\begin{array}{c}\overline{75 \cdot 3} \\
\overline{76 \cdot 4} \\
76 \cdot 5\end{array}$ & $\begin{array}{l}\overline{1 \cdot 84} \\
\overline{1 \cdot 50} \\
1 \cdot 71\end{array}$ & $\begin{array}{l}8 \cdot 99 \\
9 \cdot 05 \\
9 \cdot 05 \\
8 \cdot 33 \\
7 \cdot 78\end{array}$ & $\begin{array}{l}+2 \cdot 52 \\
+0.62 \\
+4 \cdot 41 \\
-0.38 \\
-1 \cdot 34\end{array}$ & $18 \cdot 53$ & $\begin{array}{l}0.5712 \\
0.4527 \\
0.4308 \\
0.5040 \\
0.5264\end{array}$ & $\begin{array}{c}-\overline{7621} \\
\overline{0} \\
0 . \overline{6220} \\
0.7061\end{array}$ & $\begin{array}{l}0.85 \\
0.85 \\
0.85 \\
0.85 \\
0.96\end{array}$ & $\begin{array}{l}+0.28 \\
-0.36 \\
+0.42 \\
-0.28 \\
-0.27\end{array}$ & $\begin{array}{c}\overline{4} \\
4 \cdot 68 \\
\overline{3 \cdot 82} \\
4 \cdot 33\end{array}$ & $\begin{array}{l}36 \cdot 98 \\
36 \cdot 98 \\
36 \cdot 98 \\
36 \cdot 96 \\
41 \cdot 05\end{array}$ & $\begin{array}{l}+36 \cdot 98 \\
+32 \cdot 30 \\
+36 \cdot 98 \\
+33 \cdot 14 \\
+36 \cdot 72\end{array}$ \\
\hline $\begin{array}{r}311 \\
62\end{array}$ & $\begin{array}{l}74 \cdot 6 \\
14 \cdot 9\end{array}$ & $76 \cdot 0$ & $\begin{array}{l}5 \cdot 05 \\
1 \cdot 01\end{array}$ & $\begin{array}{r}43 \cdot 20 \\
8 \cdot 64\end{array}$ & $\begin{array}{l}+5 \cdot 83 \\
+1 \cdot 17\end{array}$ & $\begin{array}{l}-170 g \\
-34 g\end{array}$ & $\begin{array}{l}2 \cdot 4851 \\
0.4970\end{array}$ & $\begin{array}{l}2 \cdot 0902 \\
0 \cdot 4180\end{array}$ & $\begin{array}{l}4 \cdot 36 \\
0 \cdot 87\end{array}$ & $\begin{array}{l}-0.21 \\
-0.04\end{array}$ & $\begin{array}{r}12 \cdot 83 \\
2 \cdot 56\end{array}$ & $\begin{array}{r}188 \cdot 95 \\
37 \cdot 59\end{array}$ & $\begin{array}{r}+176 \cdot 12 \\
+35 \cdot 03\end{array}$ \\
\hline $\begin{array}{l}\overline{76} \\
\overline{96} \\
\overline{9} \\
94\end{array}$ & $\begin{array}{l}\overline{16 \cdot 8} \\
\overline{20 \cdot 5} \\
\overline{20 \cdot 0} \\
21 \cdot 1\end{array}$ & $\begin{array}{l}\overline{78 \cdot 9} \\
\overline{78 \cdot 6} \\
\overline{77 \cdot 8} \\
77 \cdot 6\end{array}$ & $\begin{array}{l}\overline{1 \cdot 03} \\
\overline{1 \cdot 27} \\
\overline{1 \cdot 24} \\
1 \cdot 31\end{array}$ & $\begin{array}{l}7 \cdot 78 \\
7 \cdot 78 \\
7 \cdot 78 \\
7 \cdot 78 \\
7 \cdot 78 \\
7 \cdot 78 \\
7 \cdot 72\end{array}$ & $\begin{array}{l}+1.12 \\
-0.19 \\
+0.94 \\
+1.25 \\
+4.60 \\
-3.73 \\
-0.75\end{array}$ & $18 \cdot 75$ & $\begin{array}{l}0.4790 \\
0.5136 \\
0.5778 \\
0.4002 \\
0.3149 \\
0.8616 \\
0.6174\end{array}$ & $\begin{array}{c}0 \cdot \overline{3861} \\
-\overline{4711} \\
0 \cdot \overline{-} \\
0 \cdot \overline{4596} \\
0 \cdot 4849\end{array}$ & $\begin{array}{l}0.96 \\
0.96 \\
0.96 \\
0.96 \\
0.96 \\
0.96 \\
0.97\end{array}$ & $\begin{array}{l}+0.48 \\
+0.06 \\
+0.38 \\
+0.09 \\
+0.65 \\
-0.36 \\
-0.13\end{array}$ & $\begin{array}{l}\overline{3 \cdot 91} \\
\overline{-} \\
4 \cdot 77 \\
\overline{4} \\
4 \cdot 97\end{array}$ & $\begin{array}{l}41 \cdot 05 \\
41 \cdot 05 \\
41 \cdot 05 \\
41 \cdot 05 \\
41 \cdot 05 \\
41 \cdot 05 \\
37 \cdot 50\end{array}$ & $\begin{array}{l}+41 \cdot 05 \\
+37 \cdot 14 \\
+41 \cdot 05 \\
+36 \cdot 28 \\
+41 \cdot 05 \\
+36 \cdot 38 \\
+32 \cdot 59\end{array}$ \\
\hline $\begin{array}{r}356 \\
51\end{array}$ & $\begin{array}{l}78 \cdot 4 \\
11 \cdot 2\end{array}$ & $77 \cdot 8$ & $\begin{array}{l}4 \cdot 85 \\
0 \cdot 69\end{array}$ & $\begin{array}{r}54 \cdot 42 \\
7 \cdot 77\end{array}$ & $\begin{array}{l}+3.26 \\
+0.47\end{array}$ & $\begin{array}{c}+390 g . \\
\text { Gain } \\
+56 g .\end{array}$ & $\begin{array}{l}3 \cdot 7645 \\
0 \cdot 5378\end{array}$ & $\begin{array}{l}1 \cdot 8017 \\
0 \cdot 2574\end{array}$ & $\begin{array}{l}6 \cdot 73 \\
0 \cdot 96\end{array}$ & $\begin{array}{l}+1 \cdot 16 \\
+0 \cdot 17\end{array}$ & $\begin{array}{r}18 \cdot 26 \\
2 \cdot 61\end{array}$ & $\begin{array}{r}283 \cdot 80 \\
40 \cdot 54\end{array}$ & $\begin{array}{r}+265 \cdot 54 \\
+37 \cdot 93\end{array}$ \\
\hline $\begin{array}{r}- \\
\overline{89} \\
55 \\
229\end{array}$ & $\begin{array}{c}- \\
20 \cdot 8 \\
12 \cdot 9 \\
34 \cdot 2\end{array}$ & $\begin{array}{l}- \\
76 \cdot 6 \\
76 \cdot 5 \\
85 \cdot 1\end{array}$ & $\begin{array}{l}- \\
\overline{1 \cdot 46} \\
0 \cdot 91 \\
2 \cdot 39\end{array}$ & $\begin{array}{l}7 \cdot 72 \\
7 \cdot 76 \\
7 \cdot 95 \\
7 \cdot 95 \\
7 \cdot 37\end{array}$ & $\begin{array}{l}+1.50 \\
+0.53 \\
+0.45 \\
+0.17 \\
-0.40\end{array}$ & $18 \cdot 78$ & $\begin{array}{l}0.4932 \\
0.6320 \\
0.5627 \\
0.6628 \\
0.4416\end{array}$ & $\begin{array}{c}0.4545 \\
-\overline{45} 45 \\
0.4545 \\
0.2819 \\
0.7472\end{array}$ & $\begin{array}{l}0.97 \\
0.97 \\
0.97 \\
0.97 \\
0.97\end{array}$ & $\begin{array}{l}+0.02 \\
+0.34 \\
-0.05 \\
+0.03 \\
-0.22\end{array}$ & $\begin{array}{l}- \\
- \\
3 \cdot 99 \\
2 \cdot 47 \\
6 \cdot 50\end{array}$ & $\begin{array}{l}37 \cdot 50 \\
37 \cdot 50 \\
43 \cdot 10 \\
43 \cdot 10 \\
43 \cdot 10\end{array}$ & $\begin{array}{l}+37 \cdot 50 \\
+37.50 \\
+39 \cdot 11 \\
+40 \cdot 63 \\
+36 \cdot 60\end{array}$ \\
\hline $\begin{array}{r}373 \\
75\end{array}$ & $\begin{array}{l}67 \cdot 9 \\
13 \cdot 6\end{array}$ & $81 \cdot 3$ & $\begin{array}{l}4 \cdot 76 \\
0 \cdot 95\end{array}$ & $\begin{array}{r}38 \cdot 75 \\
7 \cdot 75\end{array}$ & $\begin{array}{l}+2 \cdot 25 \\
+0.45\end{array}$ & $\begin{array}{l}+30 g . \\
\text { Gain } \\
+6 g .\end{array}$ & $\begin{array}{l}2 \cdot 7923 \\
0 \cdot 5584\end{array}$ & $\begin{array}{l}1 \cdot 9381 \\
0 \cdot 3876\end{array}$ & $\begin{array}{l}4 \cdot 85 \\
0 \cdot 97\end{array}$ & $\begin{array}{l}+0.12 \\
+0.02\end{array}$ & $\begin{array}{r}12 \cdot 96 \\
2 \cdot 59\end{array}$ & $\begin{array}{r}204 \cdot 30 \\
40 \cdot 87\end{array}$ & $\begin{array}{r}+191 \cdot 34 \\
+38 \cdot 27\end{array}$ \\
\hline $\begin{array}{l}\overline{117} \\
132 \\
\overline{121}\end{array}$ & $\begin{array}{l}-\overline{24 \cdot 1} \\
13 \cdot 0 \\
\overline{29 \cdot 6}\end{array}$ & & $\begin{array}{l}\overline{1 \cdot 65} \\
0 \cdot 89 \\
\overline{2 \cdot 04}\end{array}$ & $\begin{array}{l}7 \cdot 39 \\
7 \cdot 97 \\
7 \cdot 97 \\
7 \cdot 97 \\
7 \cdot 97\end{array}$ & $\begin{array}{l}+2.18 \\
-0.09 \\
+0.29 \\
+0.89 \\
-0.56\end{array}$ & $18 \cdot 78$ & $\begin{array}{l}0 \cdot 5674 \\
0 \cdot 4702 \\
0 \cdot 4211 \\
0.5966 \\
0.5685\end{array}$ & $\begin{array}{c}-\overline{5644} \\
0.5044 \\
0.304 \\
\overline{0.6932}\end{array}$ & $\begin{array}{l}0.97 \\
0.97 \\
0.96 \\
0.96 \\
0.96\end{array}$ & $\begin{array}{l}+0.40 \\
-0.06 \\
+0.23 \\
+0.36 \\
-0.30\end{array}$ & $\begin{array}{l}- \\
3 \cdot 54 \\
1 \cdot 91 \\
- \\
4 \cdot 34\end{array}$ & $\begin{array}{l}43 \cdot 36 \\
43 \cdot 36 \\
43 \cdot 27 \\
43 \cdot 27 \\
43 \cdot 27\end{array}$ & $\begin{array}{l}+43 \cdot 36 \\
+39 \cdot 82 \\
+41 \cdot 36 \\
+43 \cdot 27 \\
+38 \cdot 93\end{array}$ \\
\hline \begin{tabular}{r|}
370 \\
74
\end{tabular} & $\begin{array}{l}66 \cdot 7 \\
13 \cdot 3\end{array}$ & $82 \cdot 0$ & $\begin{array}{l}4 \cdot \tilde{\text { อे }} \\
0 \cdot 92\end{array}$ & $\begin{array}{r}39 \cdot 27 \\
7 \cdot 85\end{array}$ & $\begin{array}{l}+2.71 \\
+0.54\end{array}$ & $\begin{array}{l}+30 g \\
\text { Gain } \\
+6 g .\end{array}$ & $\begin{array}{l}2 \cdot 6238 \\
0.5247\end{array}$ & $\begin{array}{l}1 \cdot 5620 \\
0.3124\end{array}$ & $\begin{array}{l}4.82 \\
0.96\end{array}$ & $\begin{array}{l}+0.64 \\
+0.13\end{array}$ & $\begin{array}{l}9 \cdot 79 \\
1 \cdot 96\end{array}$ & $\begin{array}{r}216 \cdot 53 \\
43 \cdot 30\end{array}$ & $\begin{array}{r}+206 \cdot 74 \\
+41 \cdot 35\end{array}$ \\
\hline
\end{tabular}


The results expressed in the above table are represented graphically in the following curves:

\section{Curve III.,}

showing the influence of boric acid and borax upon nitrogen and phosphorus metabolism, etc.

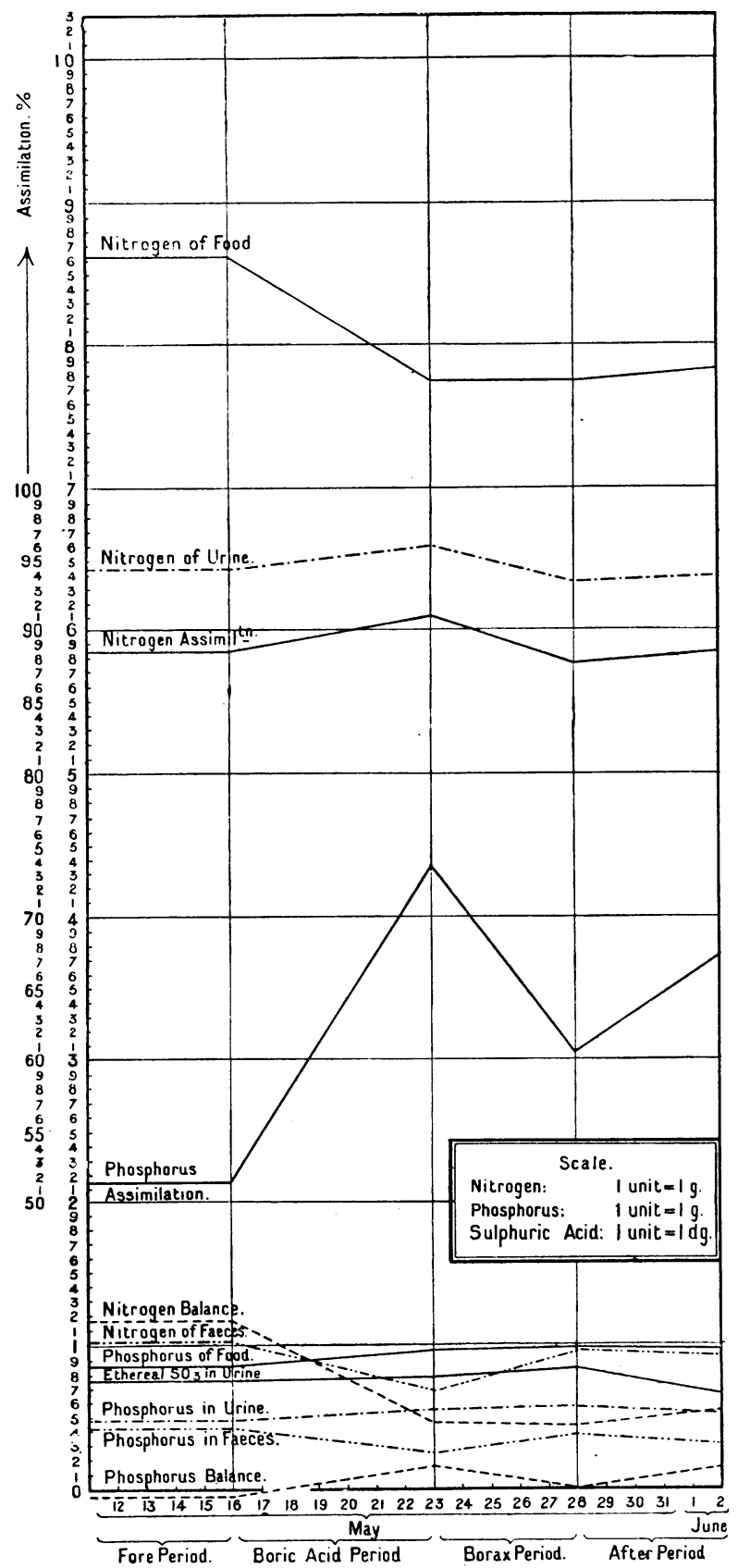


Curve IV.,

showing the influence of boric acid and borax upon fat metabolism and the quantity of faeces and urine.

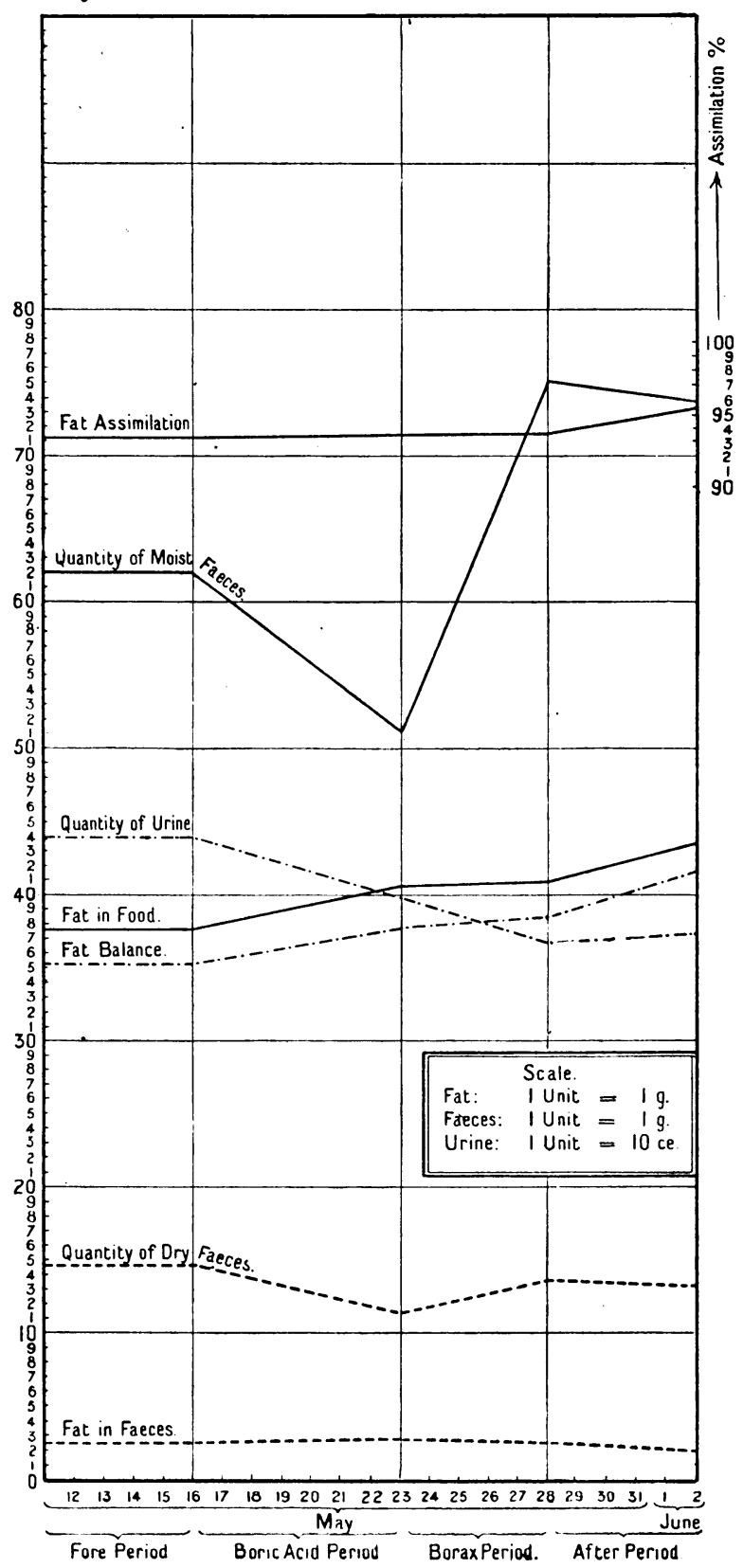


Adopting the same method as in the previous observation we arrive at the following results :-

Nitrogen Metabolism.

\begin{tabular}{|c|c|c|c|c|c|c|}
\hline$\ldots$ & & & Fore period & $\begin{array}{l}\text { Boric acid } \\
\text { period }\end{array}$ & Borax period & After period \\
\hline $\begin{array}{c}\text { Nitrogen in } \text { Food ... } \\
\text {, } \quad \text {, Urine } \\
, \quad \text {, Faeces }\end{array}$ & $\begin{array}{l}\cdots \\
\cdots \\
\cdots\end{array}$ & $\begin{array}{l}\ldots \\
. . \\
\ldots\end{array}$ & $\begin{array}{l}8 \cdot 64 \\
6 \cdot 46 \\
1 \cdot 01\}\end{array}$ & $\left.\begin{array}{l}7 \cdot 77 \\
6 \cdot 61 \\
0 \cdot 69\end{array}\right\}$ & $\begin{array}{l}7 \cdot 75 \\
6 \cdot 35\} \\
0 \cdot 95\}\end{array}$ & $\left.\begin{array}{l}7 \cdot 85 \\
6 \cdot 39 \\
0 \cdot 92\end{array}\right\}$ \\
\hline 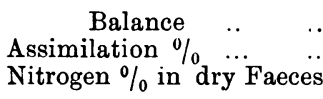 & $\begin{array}{l}\cdots \\
\cdots \\
\text { es }\end{array}$ & \begin{tabular}{l|}
$\cdots$ \\
$\cdots$ \\
$\cdots$
\end{tabular} & $\begin{array}{c}+1 \cdot 17 \\
88 \cdot 31 \\
6 \cdot 7\end{array}$ & $\begin{array}{c}+0 \cdot 47 \\
91 \cdot 12 \\
6 \cdot 1\end{array}$ & $\begin{array}{c}+0 \cdot 45 \\
87 \cdot 74 \\
6 \cdot 9\end{array}$ & $\begin{array}{c}+0 \cdot 54 \\
88 \cdot 28 \\
6 \cdot 9\end{array}$ \\
\hline
\end{tabular}

From these figures it will be seen that the nitrogen metabolism underwent no change. The assimilation of the nitrogenous food was improved during the boric acid period, and practically not affected during the borax period. The tendency of the body to reach equilibrium is clearly seen in the balance figures. The variation of the nitrogen in the urine during the respective periods is very small, but may be regarded as pointing to a slight stimulation of proteid katabolism during the boric acid period, and the reverse during the borax period.

Phosphorus Metabolism.

\begin{tabular}{|c|c|c|c|c|c|c|c|}
\hline & & & & Fore period & $\begin{array}{l}\text { Boric acid } \\
\text { period }\end{array}$ & Borax period & After period \\
\hline $\begin{array}{c}\text { Phosphorus i } \\
, ",\end{array}$ & $\begin{array}{l}\text { in Food } \\
\text {, Urine } \\
\text {, Faeces }\end{array}$ & $\begin{array}{l}\cdots \\
\cdots \\
\cdots\end{array}$ & $\begin{array}{l}\cdots \\
\cdots \\
\cdots\end{array}$ & $\left.\begin{array}{l}0 \cdot 87 \\
0 \cdot 4970 \\
0 \cdot 4180\end{array}\right\}$ & $\left.\begin{array}{l}0 \cdot 96 \\
0 \cdot 5378 \\
0 \cdot 2574\end{array}\right\}$ & $\left.\begin{array}{l}0 \cdot 97 \\
0 \cdot 5584 \\
0 \cdot 3876\end{array}\right\}$ & $\left.\begin{array}{l}0.96 \\
0.5247 \\
0 \cdot 3124\end{array}\right\}$ \\
\hline $\begin{array}{l}\text { Balanc } \\
\text { Assimilation } \\
\text { Phosphorus } 0\end{array}$ & 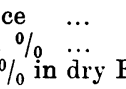 & $\begin{array}{c}\ldots \\
\ldots \\
\text { aeces }\end{array}$ & $\begin{array}{l}\cdots \\
\cdots \\
\cdots\end{array}$ & $\begin{array}{c}-0 \cdot 04 \\
51 \cdot 72 \\
2 \cdot 9\end{array}$ & $\begin{array}{c}+0 \cdot 17 \\
73 \cdot 30 \\
2 \cdot 3\end{array}$ & $\begin{array}{c}+0 \cdot 02 \\
60 \cdot 04 \\
2 \cdot 8\end{array}$ & $\begin{array}{c}+0 \cdot 13 \\
67 \cdot 46 \\
2 \cdot 3\end{array}$ \\
\hline
\end{tabular}

From the above figures it will be seen that these drugs did not affect the phosphorus metabolism, but that the assimilation of phosphorus was rather improved by them, especially by boric acid. Taking into consideration the fact that the phosphorus in the food was increased during the drug periods, the slight increase of phosphorus in the urine cannot be regarded as pointing to an increased phosphorus katabolism. 
Fat Assimilation.

\begin{tabular}{|c|c|c|c|c|c|c|c|}
\hline & & . & & Fore period & $\begin{array}{c}\text { Boric acid } \\
\text { period }\end{array}$ & Borax period & After period \\
\hline $\begin{array}{l}\text { Fat in Food } \\
\quad,, \quad \text { Faeces }\end{array}$ & $\begin{array}{l}\cdots \\
\cdots\end{array}$ & $\begin{array}{l}\cdots \\
\cdots\end{array}$ & $\begin{array}{l}\cdots \\
\cdots\end{array}$ & $\begin{array}{r}37 \cdot 59 \\
2 \cdot 56\end{array}$ & $\begin{array}{r}40 \cdot 54 \\
2 \cdot 61\end{array}$ & $\begin{array}{r}40 \cdot 87 \\
2 \cdot 59\end{array}$ & $\begin{array}{r}43 \cdot 30 \\
1 \cdot 96\end{array}$ \\
\hline \begin{tabular}{l}
\multicolumn{1}{c}{ Balance } \\
Assimilation $\%$ \\
Fat in dry Faeces
\end{tabular} & $\begin{array}{l}\cdots \\
\ldots \%\end{array}$ & $\begin{array}{l}\cdots \\
\cdots \\
\cdots\end{array}$ & $\begin{array}{l}\cdots \\
\cdots \\
\cdots\end{array}$ & $\begin{array}{c}+35 \cdot 03 \\
93 \cdot 19 \\
17 \cdot 2\end{array}$ & $\begin{array}{c}+37 \cdot 93 \\
93 \cdot 57 \\
23 \cdot 3\end{array}$ & $\begin{array}{c}+38 \cdot 27 \\
93 \cdot 66 \\
19 \cdot 0\end{array}$ & $\begin{array}{c}+41 \cdot 35 \\
95 \cdot 47 \\
14 \cdot 7\end{array}$ \\
\hline
\end{tabular}

From the figures in this table it will be seen that the same remarks apply as in Observation I., viz. that boric acid and borax exerted no influence upon fat assimilation.

The remaining points to be considered may, as in the preceding observation, be divided as follows:

The quantity of urine was decidedly diminished during the borax period, to a less extent during the boric acid period. The specific gravity increased with the diminishing volume. The reaction of the urine kept constantly acid to litmus during both the boric acid and borax period. In the fore period it varied between amphoteric and acid.

Quantity of faeces. During the boric acid period the quantity of faeces was slightly decreased.

The uric acid variation is too slight to permit of any conclusion being drawn from it. The alteration in the quantity of total sulphuric acid during the respective periods was very slight, but in the same direction as that of the total nitrogen. The ethereal sulphates underwent no change during the boric acid period, but increased slightly during the borax period. Neither substance exerted therefore any intestinal antiseptic action, the increase during the borax period is probably an alkali effect, the same having been observed in the case of other alkaline salts. Boric acid showed itself in the urine of the first day of its administration, and disappeared completely in the course of the second day of the after period.

The body weight increased during the boric acid and borax periods.

The results relevant to the observations made above are summarised in the following table:- 
TABLE III A.

\begin{tabular}{|c|c|c|c|c|c|c|c|c|}
\hline & $\begin{array}{c}\text { Nitrogen } \\
\text { assimilation } \\
\%\end{array}$ & $\begin{array}{l}\% / 0 \mathrm{~N} . \\
\text { of dry } \\
\text { faeces }\end{array}$ & $\begin{array}{l}\text { Phosphorus } \\
\text { assimilation } \\
\% \%\end{array}$ & $\begin{array}{l}\% \% \mathrm{P} . \\
\text { of dry } \\
\text { faeces }\end{array}$ & $\underset{0 \%}{\text { Fat }}$ & $\begin{array}{l}\% \text { fat } \\
\text { of dry } \\
\text { faeces }\end{array}$ & $\frac{\mathbf{A}^{*}}{\mathbf{B}}$ & $\frac{\mathrm{N} \dagger}{\mathrm{SO}_{3}}$ \\
\hline Fore period & $88 \cdot 31$ & $6 \cdot 7$ & $51 \cdot 72$ & $2 \cdot 9$ & $93 \cdot 19$ & $17 \cdot 2$ & $15 \cdot 2$ & $5 \cdot 1$ \\
\hline Boric acid period & $91 \cdot 12$ & $6 \cdot 1$ & $73 \cdot 30$ & $2 \cdot 3$ & $93 \cdot 57$ & $23 \cdot 3$ & $15 \cdot 0$ & $5 \cdot 3$ \\
\hline Borax period ... & $87 \cdot 74$ & $6 \cdot 9$ & $60 \cdot 04$ & $2 \cdot 8$ & $93 \cdot 66$ & $19 \cdot 0$ & $13 \cdot 7$ & $5 \cdot 3$ \\
\hline After period & $88 \cdot 28$ & $6 \cdot 9$ & $67 \cdot 46$ & $2 \cdot 3$ & $95 \cdot 47$ & $14 \cdot 7$ & $17 \cdot 5$ & $5 \cdot 5$ \\
\hline
\end{tabular}

* As in Table II A.

+ As in Table II $\mathrm{A}$.

\section{Observation III. Child C.}

The child was a delicate girl, aged four years, weighing $15 \cdot 6$ kilos. She was convalescent from pneumonia and compared with the other children not so well nourished or developed. She consumed daily $200 \mathrm{~g}$. of bread, 550 c.c. of milk, $20 \mathrm{~g}$. of butter, $30 \mathrm{~g}$. of meat, $50 \mathrm{~g}$. of apple compote, $10 \mathrm{~g}$. of sugar, 50 c.c. of water, $5 \mathrm{~g}$. of toffee.

The whole observation lasted for 22 days, of which five days were devoted to the fore period, seven days to boric acid, 5 days to borax, and 5 days to the after period.

The boric acid and borax were administered as shown in the following table:

Boric Acid Period.

3 days: 0.5 g. per diem $=1$ in 1000 in Milk $=1$ in 1800 in total Food and Drink.

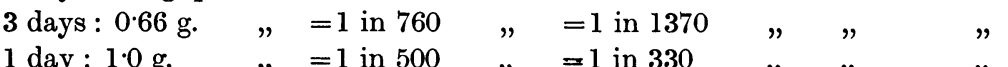

Borax Period.

5 days : $1.5 \mathrm{~g} . \quad, \quad=1$ in $330 \quad,=1$ in $600 \quad, \quad, \quad$,

It may be noted that the maximum medicinal dose for this child is in the case of boric acid 0.24 g., of borax $0.33 \mathrm{~g}$., and that the quantities given as in the former observations, are greatly in excess of those which would be required as a food preservative.

The analytical results obtained throughout this observation are recorded in Table IV. p. 192 :-

Adopting the same method as in the previous observations we arrive at the following results with regard to,

Nitrogen Metabolism.

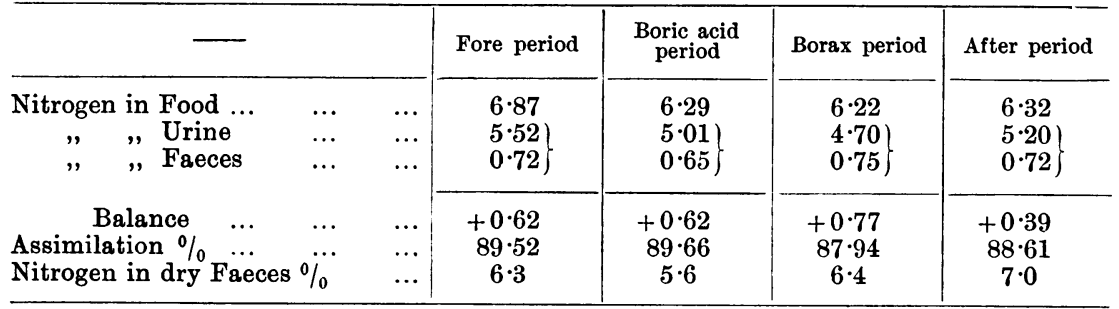


The assimilation of proteids was in this case not affected by boric acid, but slightly decreased by borax. The balance remained practically constant, being near the equilibrium.

In this case boric acid does not seem to have stimulated proteid katabolism, whilst borax showed its usual inhibitory tendency.

Phosphorus Metabolism.

\begin{tabular}{|c|c|c|c|c|c|c|c|}
\hline & \multicolumn{3}{|l|}{$\longrightarrow$} & \multirow{2}{*}{$\begin{array}{c}\text { Fore period } \\
\begin{array}{l}0.78 \\
0 \cdot 4399 \\
0 \cdot 2772\}\end{array}\end{array}$} & \multirow{2}{*}{$\left.\begin{array}{l}\begin{array}{c}\text { Boric acid } \\
\text { period }\end{array} \\
0 \cdot 80 \\
0 \cdot 4186 \\
0.2406\end{array}\right\}$} & \multirow{2}{*}{$\begin{array}{l}\text { Borax period } \\
\left.\begin{array}{l}0 \cdot 81 \\
0 \cdot 4168 \\
0 \cdot 2655\end{array}\right\}\end{array}$} & \multirow{2}{*}{$\begin{array}{c}\text { After period } \\
0 \cdot 80 \\
\left.\begin{array}{l}0.4619 \\
0 \cdot 2410\end{array}\right\}\end{array}$} \\
\hline $\begin{array}{c}\text { Phosphorus in } \\
, "\end{array}$ & $\begin{array}{l}\text { Food } \\
\text { Urine } \\
\text { Faeces }\end{array}$ & $\begin{array}{l}\cdots \\
\cdots \\
\cdots\end{array}$ & $\begin{array}{l}\cdots \\
\cdots \\
\cdots\end{array}$ & & & & \\
\hline $\begin{array}{l}\text { Balance } \\
\text { Assimilation } \% \\
\text { Phosphorus in }\end{array}$ & $\begin{array}{c}\ldots \\
\ldots \\
\text { dry Fae }\end{array}$ & $\begin{array}{l}\cdots \\
\cdots \\
e s\end{array}$ & $\begin{array}{l}\cdots \\
\cdots \\
\cdots\end{array}$ & $\begin{array}{c}+0 \cdot 06 \\
64 \cdot 46 \\
2 \cdot 4\end{array}$ & $\begin{array}{c}+0 \cdot 14 \\
70 \cdot 00 \\
2 \cdot 1\end{array}$ & $\begin{array}{c}+0 \cdot 13 \\
67 \cdot 23 \\
2 \cdot 1\end{array}$ & $\begin{array}{c}+0 \cdot 10 \\
69 \cdot 88 \\
2 \cdot 3\end{array}$ \\
\hline
\end{tabular}

As in the former cases the phosphorus assimilation was improved, especially in the boric acid period. The katabolism of substances rich in phosphorus seemed to be slightly inhibited in both periods.

Fat Assimilation.

\begin{tabular}{cccc|c|c|c|c}
\hline & & & & Fore period & $\begin{array}{c}\text { Boric acid } \\
\text { period }\end{array}$ & Borax period & After period \\
\hline Fat in Food & $\ldots$ & $\ldots$ & $\ldots$ & $35 \cdot 50$ & $37 \cdot 16$ & $39 \cdot 00$ & $41 \cdot 68$ \\
,, Faeces & $\ldots$ & $\ldots$ & $\ldots$ & $2 \cdot 35$ & $2 \cdot 44$ & $2 \cdot 48$ & $1 \cdot 80$ \\
\hline Balance & $\ldots$ & $\ldots$ & $\ldots$ & $+33 \cdot 14$ & $+34 \cdot 71$ & $+36 \cdot 51$ & $+39 \cdot 88$ \\
Assimilation $\%$ & $\ldots$ & $\ldots$ & $\ldots$ & $93 \cdot 38$ & $93 \cdot 43$ & $93 \cdot 64$ & $95 \cdot 68$ \\
Fat in dry Faeces $\%$ & $\ldots$ & $\ldots$ & $20 \cdot 6$ & $20 \cdot 8$ & $21 \cdot 2$ & $17 \cdot 8$ \\
\hline
\end{tabular}

As in the former cases the fat balance increased with the amount of fat ingested. The assimilation of fat was not affected.

The remaining points to be considered may as in the preceding observations be classified as follows:

The quantity of urine decreased during the boric acid and borax period to the same extent, the specific gravity increasing with the diminishing volume. The reaction varied between acid and amphoteric during the boric acid period, and remained acid throughout the borax period.

The quantity of dry faeces underwent no change during the boric acid and borax period. 
192 Influence of Boric Acid and Borax, etc.

\section{TABLE IV.}

Showing the Influence of Boric Acid and Borax upon

\begin{tabular}{|c|c|c|c|c|c|c|c|c|c|c|}
\hline \multirow[b]{2}{*}{ PERIOD } & & \multirow[b]{2}{*}{ Date } & \multirow[b]{2}{*}{$\begin{array}{c}\text { Dose } \\
\mathrm{g} \\
\end{array}$} & \multicolumn{7}{|c|}{ URINE } \\
\hline & & & & $\begin{array}{c}\text { Quantity } \\
\text { c.c. }\end{array}$ & Reaction & $\begin{array}{l}\text { Specific } \\
\text { gravity }\end{array}$ & $\begin{array}{c}\text { Total } \\
\text { sulphuric } \\
\text { acid } \\
\mathrm{g}\end{array}$ & $\begin{array}{c}\text { Ethereal } \\
\text { sulphuric } \\
\text { acid } \\
\text { g }\end{array}$ & $\begin{array}{l}\text { Uric } \\
\text { acid } \\
\mathrm{g}\end{array}$ & $\begin{array}{c}\text { Nitrogen } \\
\mathrm{g} \\
\end{array}$ \\
\hline \multirow[t]{2}{*}{$\begin{array}{c}\text { FORE } \\
\text { PERIOD }\end{array}$} & & $\begin{array}{l}12 \mathrm{~V} . \\
13,, \\
14,, \\
15,, \\
16,,\end{array}$ & & $\begin{array}{l}445 \\
400 \\
480 \\
350 \\
275\end{array}$ & $\begin{array}{l}\text { Amphoteric } \\
\text { Acid } \\
\text { Amphoteric }\end{array}$ & $\begin{array}{l}1 \cdot 0200 \\
1 \cdot 0226 \\
1 \cdot 0200 \\
1 \cdot 0227 \\
1 \cdot 0270\end{array}$ & $\begin{array}{l}1 \cdot 1861 \\
1 \cdot 0671 \\
1 \cdot 2805 \\
0 \cdot 9337 \\
0 \cdot 7336\end{array}$ & $\begin{array}{l}0 \cdot 0719 \\
0 \cdot 0646 \\
0 \cdot 0776 \\
0 \cdot 0566 \\
0 \cdot 0444\end{array}$ & $\begin{array}{l}0 \cdot 1902 \\
0 \cdot 1710 \\
0 \cdot 2052 \\
0 \cdot 1496 \\
0 \cdot 1176\end{array}$ & $\begin{array}{l}5 \cdot 74 \\
5 \cdot 72 \\
6 \cdot 48 \\
5 \cdot 09 \\
4 \cdot 59\end{array}$ \\
\hline & $\begin{array}{l}\text { Total } \\
\text { Average }\end{array}$ & $\begin{array}{l}5 \text { days } \\
1 \text { day }\end{array}$ & & $\begin{array}{r}1950 \\
390\end{array}$ & & $1 \cdot 0224$ & $\begin{array}{l}5 \cdot 2010 \\
1 \cdot 0402\end{array}$ & $\begin{array}{l}0 \cdot 1151 \\
0 \cdot 0630\end{array}$ & $\begin{array}{l}0 \cdot 8336 \\
0 \cdot 1667\end{array}$ & $\begin{array}{r}27 \cdot 62 \\
5 \cdot 52\end{array}$ \\
\hline \multirow[t]{2}{*}{$\begin{array}{l}\text { BORIC ACID } \\
\text { PERIOD }\end{array}$} & & $\begin{array}{l}17 \mathrm{~V} . \\
18,, \\
19 \quad, \\
20, \\
21, \\
22,, \\
23 \quad,\end{array}$ & $\begin{array}{l}0.50 \\
0.50 \\
0.50 \\
0.66 \\
0.66 \\
0.66 \\
1.00\end{array}$ & $\begin{array}{l}405 \\
320 \\
330 \\
260 \\
310 \\
250 \\
200\end{array}$ & $\begin{array}{c}\text { Acid } \\
\text {," } \\
\text {,' } \\
\text { Amphoteric } \\
\text { Acid }\end{array}$ & $\begin{array}{l}1 \cdot 0200 \\
1 \cdot 0285 \\
1 \cdot 0269 \\
1 \cdot 0275 \\
1 \cdot 0260 \\
1 \cdot 0295 \\
1 \cdot 0255\end{array}$ & $\begin{array}{l}1 \cdot 2422 \\
0 \cdot 9816 \\
0 \cdot 8589 \\
0 \cdot 9662 \\
0 \cdot 7208 \\
0 \cdot 8580 \\
0 \cdot 6864\end{array}$ & $\begin{array}{l}0.0818 \\
0.0646 \\
0.0667 \\
0.0525 \\
0.0626 \\
0.0590 \\
0.0472\end{array}$ & $\begin{array}{l}0 \cdot 1124 \\
0 \cdot 0888 \\
0 \cdot 0916 \\
0 \cdot 0722 \\
0 \cdot 0860 \\
0 \cdot 1444 \\
0 \cdot 1155 \\
\end{array}$ & $\begin{array}{l}5 \cdot 18 \\
5 \cdot 93 \\
5 \cdot 46 \\
4 \cdot 75 \\
5 \cdot 40 \\
4 \cdot 91 \\
3 \cdot 44\end{array}$ \\
\hline & $\begin{array}{l}\text { Total } \\
\text { Average }\end{array}$ & $\begin{array}{l}7 \text { days } \\
1 \text { day }\end{array}$ & $\begin{array}{l}4 \cdot 48 \\
0 \cdot 64\end{array}$ & $\begin{array}{r}2075 \\
296\end{array}$ & & $1 \cdot 0262$ & $\begin{array}{l}6 \cdot 3141 \\
0 \cdot 9020\end{array}$ & $\begin{array}{c}0.4344 \\
0 \cdot 0620\end{array}$ & $\begin{array}{l}0 \cdot 7109 \\
0 \cdot 1015\end{array}$ & $\begin{array}{r}35 \cdot 07 \\
5 \cdot 01\end{array}$ \\
\hline \multirow[t]{2}{*}{$\begin{array}{c}\text { BORAX } \\
\text { PERIOD }\end{array}$} & & $\begin{array}{ll}24 & \mathrm{~V} . \\
25 & , \\
26 & , \\
27 & , \\
28 & , \\
\end{array}$ & $\begin{array}{l}1.5 \\
1.5 \\
1.5 \\
1.5 \\
1.5\end{array}$ & $\begin{array}{l}355 \\
320 \\
280 \\
315 \\
235\end{array}$ & $\begin{array}{c}\text { Acid } \\
\text {," } \\
,, \\
,, \\
\end{array}$ & $\begin{array}{l}1 \cdot 0236 \\
1 \cdot 0215 \\
1 \cdot 0260 \\
1 \cdot 0247 \\
1 \cdot 0276 \\
\end{array}$ & $\begin{array}{l}1.0438 \\
0.9409 \\
0.8229 \\
0.9267 \\
0.6909\end{array}$ & $\begin{array}{l}0 \cdot 0774 \\
0 \cdot 0698 \\
0 \cdot 0610 \\
0 \cdot 0686 \\
0 \cdot 0512 \\
\end{array}$ & $\begin{array}{l}0 \cdot 2103 \\
0 \cdot 1896 \\
0 \cdot 1659 \\
0 \cdot 1866 \\
0 \cdot 1392 \\
\end{array}$ & $\begin{array}{l}5 \cdot 33 \\
4 \cdot 39 \\
4 \cdot 73 \\
4 \cdot 76 \\
4 \cdot 29 \\
\end{array}$ \\
\hline & $\begin{array}{l}\text { Total } \\
\text { Average }\end{array}$ & $\begin{array}{l}5 \text { days } \\
1 \text { day }\end{array}$ & $\begin{array}{l}7 \cdot 5 \\
1 \cdot 5\end{array}$ & $\begin{array}{r}1505 \\
301\end{array}$ & & $1 \cdot 0247$ & $\begin{array}{l}4 \cdot 4252 \\
0 \cdot 8850\end{array}$ & $\begin{array}{l}0.3280 \\
0 \cdot 0656\end{array}$ & $\begin{array}{l}0.8916 \\
0 \cdot 1783\end{array}$ & $\begin{array}{r}23 \cdot 50 \\
4 \cdot 70\end{array}$ \\
\hline \multirow[t]{2}{*}{$\begin{array}{c}\text { AFTER } \\
\text { PERIOD }\end{array}$} & & $\begin{array}{rl}29 & \mathrm{~V} . \\
30 & , \\
31 & , \\
1 & \text { VI. } \\
2 & ,, \\
\end{array}$ & & $\begin{array}{l}410 \\
365 \\
270 \\
415 \\
295\end{array}$ & $\begin{array}{c}\text { Acid } \\
,, \\
, \\
, \\
,\end{array}$ & $\begin{array}{l}1 \cdot 0193 \\
1 \cdot 0236 \\
1 \cdot 0227 \\
1 \cdot 0187 \\
1 \cdot 0270\end{array}$ & $\begin{array}{l}1 \cdot 1572 \\
1 \cdot 0302 \\
0 \cdot 7620 \\
1 \cdot 1713 \\
0 \cdot 8326\end{array}$ & $\begin{array}{l}0 \cdot 0683 \\
0 \cdot 0608 \\
0 \cdot 0450 \\
0 \cdot 0691 \\
0 \cdot 0492\end{array}$ & $\begin{array}{l}0 \cdot 0738 \\
0 \cdot 0657 \\
0 \cdot 0486 \\
0 \cdot 0747 \\
0 \cdot 0531\end{array}$ & $\begin{array}{l}5 \cdot 51 \\
5 \cdot 76 \\
4 \cdot 53 \\
5 \cdot 11 \\
5 \cdot 12\end{array}$ \\
\hline & $\begin{array}{l}\text { Total } \\
\text { Average }\end{array}$ & $\begin{array}{l}5 \text { days } \\
1 \text { day }\end{array}$ & & $\begin{array}{r}1755 \\
351\end{array}$ & & $1 \cdot 0222$ & $\begin{array}{l}4 \cdot 9533 \\
0 \cdot 9906\end{array}$ & $\begin{array}{l}0.2924 \\
0.0585\end{array}$ & $\begin{array}{l}0 \cdot 3159 \\
0 \cdot 0632\end{array}$ & $\begin{array}{r}26 \cdot 03 \\
5 \cdot 20\end{array}$ \\
\hline
\end{tabular}




\section{Table IV.}

the General Metabolism of the invalid Child C.

\begin{tabular}{|c|c|c|c|c|c|c|c|c|c|c|c|c|c|}
\hline \multicolumn{4}{|c|}{ FAECES } & \multirow[b]{2}{*}{$\begin{array}{c}\begin{array}{c}\text { Nitro- } \\
\text { gen of } \\
\text { food }\end{array} \\
\mathrm{g}\end{array}$} & \multirow[b]{2}{*}{$\begin{array}{l}\text { Balance } \\
\mathrm{g}\end{array}$} & \multirow[b]{2}{*}{$\begin{array}{r}\begin{array}{c}\text { Body } \\
\text { weight }\end{array} \\
\text { kg } \\
\end{array}$} & \multicolumn{4}{|c|}{ PHOSPHORUS } & \multicolumn{3}{|c|}{ FAT } \\
\hline $\begin{array}{c}\text { Moist } \\
\mathrm{g} \\
\end{array}$ & $\begin{array}{c}\text { Dry } \\
\mathbf{g} \\
\end{array}$ & $\begin{array}{c}\text { Water } \\
\%\end{array}$ & $\begin{array}{c}\text { Nitro- } \\
\text { gen } \\
\mathrm{g} \\
\end{array}$ & & & & $\begin{array}{c}\text { Urine } \\
\mathrm{g} \\
\end{array}$ & $\begin{array}{c}\text { Faeces } \\
\mathrm{g} \\
\end{array}$ & $\begin{array}{c}\text { Food } \\
\mathrm{g} \\
\end{array}$ & $\begin{array}{c}\text { Balance } \\
\mathrm{g}\end{array}$ & $\begin{array}{c}\text { Faeces } \\
\mathbf{g} \\
\end{array}$ & $\begin{array}{c}\text { Food } \\
\mathrm{g} \\
\end{array}$ & $\begin{array}{c}\text { Balance } \\
\mathbf{g} \\
\end{array}$ \\
\hline $\begin{array}{r}26 \\
-69 \\
48 \\
109\end{array}$ & $\begin{array}{r}7 \cdot 3 \\
15 \cdot 6 \\
9 \cdot 6 \\
24 \cdot 8\end{array}$ & $\begin{array}{c}71 \cdot 2 \\
-- \\
77 \cdot 4 \\
80 \cdot 0 \\
77 \cdot 2\end{array}$ & $\begin{array}{c}0 \cdot 46 \\
-. \\
0 \cdot 99 \\
0 \cdot 61 \\
1 \cdot 57\end{array}$ & $\begin{array}{l}7 \cdot 17 \\
7 \cdot 23 \\
7 \cdot 23 \\
6 \cdot 46 \\
6 \cdot 28\end{array}$ & $\begin{array}{r}+0.91 \\
+1.59 \\
-0.24 \\
+0.76 \\
+0.12\end{array}$ & $15 \cdot 62$ & $\begin{array}{l}0 \cdot 5020 \\
0 \cdot 4512 \\
0 \cdot 5414 \\
0 \cdot 3948 \\
0 \cdot 3102\end{array}$ & $\begin{array}{c}0 \cdot 1785 \\
- \\
0 \cdot 3814 \\
0 \cdot 2347 \\
0 \cdot 5915\end{array}$ & $\begin{array}{l}0 \cdot 76 \\
0 \cdot 76 \\
0 \cdot 76 \\
0 \cdot 81 \\
0 \cdot 80\end{array}$ & $\begin{array}{l}+0.09 \\
+0.31 \\
-0.16 \\
+0.18 \\
-0.10\end{array}$ & $\begin{array}{l}1 \cdot 50 \\
- \\
3 \cdot 21 \\
1 \cdot 97 \\
5 \cdot 10\end{array}$ & $\begin{array}{l}34 \cdot 96 \\
34 \cdot 96 \\
34 \cdot 96 \\
35 \cdot 16 \\
37 \cdot 46\end{array}$ & $\begin{array}{l}+33 \cdot 46 \\
+34 \cdot 96 \\
+31 \cdot 75 \\
+33 \cdot 19 \\
+32 \cdot 36\end{array}$ \\
\hline $\begin{array}{r}252 \\
50\end{array}$ & $\begin{array}{l}57 \cdot 3 \\
11 \cdot 4\end{array}$ & $\begin{array}{c}- \\
77 \cdot 3\end{array}$ & $\begin{array}{l}3 \cdot 63 \\
0 \cdot 72\end{array}$ & $\begin{array}{r}34 \cdot 37 \\
6 \cdot 87\end{array}$ & $\begin{array}{l}+3 \cdot 12 \\
+0 \cdot 62\end{array}$ & $\begin{array}{l}15 \cdot 62 \\
\pm 0\end{array}$ & $\begin{array}{l}2 \cdot 1996 \\
0 \cdot 4399\end{array}$ & $\begin{array}{l}1 \cdot 3861 \\
0 \cdot 2772\end{array}$ & $\begin{array}{l}3 \cdot 89 \\
0 \cdot 78\end{array}$ & $\begin{array}{l}+0.32 \\
+0.06\end{array}$ & $\begin{array}{r}11 \cdot 78 \\
2 \cdot 35\end{array}$ & $\begin{array}{l}177 \cdot 50 \\
35 \cdot 50\end{array}$ & $\begin{array}{r}+165 \cdot 72 \\
+33 \cdot 14\end{array}$ \\
\hline $\begin{array}{r}-\overline{165} \\
127 \\
9 \\
112 \\
68 \\
104\end{array}$ & $\begin{array}{r}- \\
18 \cdot 8 \\
11 \cdot 5 \\
1 \cdot 6 \\
16 \cdot 5 \\
12 \cdot 7 \\
20 \cdot 6\end{array}$ & $\begin{array}{c}- \\
88 \cdot 6 \\
90 \cdot 9 \\
82 \cdot 2 \\
85 \cdot 3 \\
81 \cdot 3 \\
80 \cdot 2\end{array}$ & $\begin{array}{l}\overline{0 \cdot 94} \\
0 \cdot 58 \\
0 \cdot 08 \\
0 \cdot 82 \\
0 \cdot 82 \\
1 \cdot 32\end{array}$ & $\begin{array}{l}6 \cdot 30 \\
6 \cdot 30 \\
6 \cdot 30 \\
6 \cdot 30 \\
6 \cdot 30 \\
6 \cdot 30 \\
6 \cdot 21\end{array}$ & $\begin{array}{l}+1.12 \\
-0.57 \\
+0.26 \\
+1.47 \\
+0.08 \\
+0.57 \\
+1.45\end{array}$ & $15 \cdot 84$ & $\begin{array}{l}0 \cdot 5464 \\
0 \cdot 4317 \\
0 \cdot 4453 \\
0 \cdot 4857 \\
0 \cdot 4182 \\
0 \cdot 3350 \\
0 \cdot 2680\end{array}$ & $\begin{array}{c}-\overline{4019} \\
0 \cdot 2458 \\
0 \cdot 0342 \\
0 \cdot 3527 \\
0 \cdot 2478 \\
0 \cdot 4019\end{array}$ & $\begin{array}{l}0 \cdot 80 \\
0 \cdot 80 \\
0 \cdot 80 \\
0 \cdot 80 \\
0 \cdot 80 \\
0 \cdot 80 \\
0 \cdot 81\end{array}$ & $\begin{array}{l}+0.25 \\
-0.03 \\
+0.11 \\
+0.28 \\
+0.03 \\
+0.22 \\
+0.14\end{array}$ & $\begin{array}{l}- \\
3 \cdot 55 \\
2 \cdot 17 \\
0 \cdot 30 \\
3 \cdot 11 \\
3 \cdot 05 \\
4 \cdot 94 \\
\end{array}$ & $\begin{array}{l}37 \cdot 46 \\
37 \cdot 46 \\
37 \cdot 46 \\
37 \cdot 46 \\
37 \cdot 46 \\
37 \cdot 46 \\
35 \cdot 37\end{array}$ & $\begin{array}{l}+37 \cdot 46 \\
+33 \cdot 91 \\
+35 \cdot 29 \\
+37 \cdot 16 \\
+34 \cdot 55 \\
+34 \cdot 41 \\
+30 \cdot 43\end{array}$ \\
\hline $\begin{array}{r}585 \\
83\end{array}$ & $\begin{array}{l}81 \cdot 7 \\
11 \cdot 7\end{array}$ & - & $\begin{array}{l}4.56 \\
0 \cdot 65\end{array}$ & $\begin{array}{r}44 \cdot 01 \\
6 \cdot 29\end{array}$ & $\begin{array}{l}+4 \cdot 38 \\
+0 \cdot 62\end{array}$ & $\begin{array}{c}+220 \\
\text { Gain } \\
+31 g .\end{array}$ & $\begin{array}{l}2 \cdot 9303 \\
0 \cdot 4186\end{array}$ & $\begin{array}{l}1 \cdot 6843 \\
0 \cdot 2406\end{array}$ & $\begin{array}{l}5 \cdot 61 \\
0 \cdot 80\end{array}$ & $\begin{array}{l}+1 \cdot 00 \\
+0 \cdot 14\end{array}$ & $\begin{array}{r}17 \cdot 12 \\
2 \cdot 44\end{array}$ & $\begin{array}{r}260 \cdot 13 \\
3 \gamma \cdot 16\end{array}$ & $\begin{array}{r}+243 \cdot 01 \\
+34 \cdot 71\end{array}$ \\
\hline $\begin{array}{r}- \\
57 \\
48 \\
64 \\
141 \\
\end{array}$ & $\begin{array}{l}- \\
13 \cdot 7 \\
10 \cdot 6 \\
12 \cdot 8 \\
21 \cdot 6\end{array}$ & $\begin{array}{l}-- \\
76 \cdot 0 \\
77 \cdot 9 \\
80 \cdot 0 \\
84 \cdot 7\end{array}$ & $\begin{array}{l}-\overline{0} \\
0 \cdot 67 \\
0 \cdot 82 \\
1 \cdot 39\end{array}$ & $\begin{array}{l}6 \cdot 21 \\
6 \cdot 25 \\
6 \cdot 41 \\
6 \cdot 41 \\
5 \cdot 84 \\
\end{array}$ & $\begin{array}{r}+0.88 \\
+0.99 \\
+1.01 \\
+0.83 \\
+0.16\end{array}$ & $16 \cdot 06$ & $\begin{array}{l}0 \cdot 4916 \\
0 \cdot 4431 \\
0 \cdot 3878 \\
0 \cdot 4362 \\
0 \cdot 3254\end{array}$ & $\begin{array}{c}-\overline{0} \cdot 3098 \\
0 \cdot 2397 \\
0 \cdot 2895 \\
0 \cdot 4885\end{array}$ & $\begin{array}{l}0 \cdot 81 \\
0 \cdot 81 \\
0 \cdot 81 \\
0 \cdot 81 \\
0 \cdot 81\end{array}$ & $\begin{array}{l}+0.32 \\
+0.06 \\
+0.18 \\
+0.09 \\
-0.01\end{array}$ & $\begin{array}{l}- \\
2 \cdot 90 \\
2 \cdot 25 \\
2 \cdot 71 \\
4 \cdot 58\end{array}$ & $\begin{array}{l}35 \cdot 37 \\
35 \cdot 37 \\
41 \cdot 42 \\
41 \cdot 42 \\
41 \cdot 42\end{array}$ & $\begin{array}{l}+35 \cdot 37 \\
+32 \cdot 47 \\
+39 \cdot 17 \\
+38 \cdot 71 \\
+36 \cdot 84\end{array}$ \\
\hline $\begin{array}{r}310 \\
62\end{array}$ & $\begin{array}{l}58 \cdot 7 \\
11 \cdot 7\end{array}$ & - & $\begin{array}{l}3 \cdot 75 \\
0 \cdot 75\end{array}$ & $\begin{array}{r}31 \cdot 12 \\
6 \cdot 22\end{array}$ & $\begin{array}{l}+3 \cdot 87 \\
+0.77\end{array}$ & $\begin{array}{l}+220 \\
\text { Gain } \\
+44 \mathrm{~g}\end{array}$ & $\begin{array}{l}2 \cdot 0841 \\
0 \cdot 4168\end{array}$ & $\begin{array}{l}1 \cdot 3275 \\
0 \cdot 2655\end{array}$ & $\begin{array}{l}4 \cdot 05 \\
0 \cdot 81\end{array}$ & $\begin{array}{l}+0 \cdot 64 \\
+0 \cdot 13\end{array}$ & $\begin{array}{r}12 \cdot 44 \\
2 \cdot 48\end{array}$ & $\begin{array}{r}195 \cdot 00 \\
39 \cdot 00\end{array}$ & $\begin{array}{r}+182 \cdot 56 \\
+36 \cdot 51\end{array}$ \\
\hline $\begin{array}{l}- \\
58 \\
90 \\
61 \\
95\end{array}$ & $\begin{array}{l}-\overline{11 \cdot 0} \\
14 \cdot 6 \\
10 \cdot 0 \\
14 \cdot 9\end{array}$ & $\begin{array}{l}-\overline{81 \cdot 0} \\
83 \cdot 0 \\
83 \cdot 6 \\
84 \cdot 3\end{array}$ & $\begin{array}{l}-\overline{0 \cdot 79} \\
1 \cdot 04 \\
0 \cdot 72 \\
1 \cdot 07\end{array}$ & $\begin{array}{l}5 \cdot 85 \\
6 \cdot 43 \\
6 \cdot 44 \\
6 \cdot 44 \\
6 \cdot 44\end{array}$ & $\begin{array}{l}+0.34 \\
-0.12 \\
+0.87 \\
+0.61 \\
+0.25\end{array}$ & $16 \cdot 00$ & $\begin{array}{l}0 \cdot 5406 \\
0 \cdot 4814 \\
0 \cdot 3502 \\
0 \cdot 5484 \\
0 \cdot 3891\end{array}$ & $\begin{array}{c}-\overline{2625} \\
0 \cdot 3484 \\
0 \cdot 2386 \\
0 \cdot 3555\end{array}$ & $\begin{array}{l}0 \cdot 81 \\
0 \cdot 81 \\
0 \cdot 80 \\
0 \cdot 80 \\
0 \cdot 80\end{array}$ & $\begin{array}{l}+0.27 \\
+0.07 \\
+0.10 \\
+0.01 \\
+0.06\end{array}$ & $\begin{array}{l}- \\
1 \cdot 96 \\
2 \cdot 61 \\
1 \cdot 78 \\
2 \cdot 65\end{array}$ & $\begin{array}{l}41 \cdot 68 \\
41 \cdot 68 \\
41 \cdot 68 \\
41 \cdot 68 \\
41 \cdot 68\end{array}$ & $\begin{array}{l}+41 \cdot 68 \\
+39 \cdot 72 \\
+39 \cdot 07 \\
+39 \cdot 90 \\
+39 \cdot 90\end{array}$ \\
\hline $\begin{array}{r}304 \\
61\end{array}$ & $\begin{array}{l}50 \cdot 5 \\
10 \cdot 1\end{array}$ & $83 \cdot 4$ & $\begin{array}{l}3 \cdot 62 \\
0 \cdot 72\end{array}$ & $\begin{array}{r}31 \cdot 60 \\
6 \cdot 32\end{array}$ & $\begin{array}{l}+1 \cdot 95 \\
+0 \cdot 39\end{array}$ & $\begin{array}{r}-60 \\
L o s s \\
-12 g\end{array}$ & $\begin{array}{l}2 \cdot 3097 \\
0 \cdot 4619\end{array}$ & $\begin{array}{l}1 \cdot 2050 \\
0 \cdot 2410\end{array}$ & $\begin{array}{l}4 \cdot 02 \\
0 \cdot 80\end{array}$ & $\begin{array}{l}+0.51 \\
+0.10\end{array}$ & $\begin{array}{l}9 \cdot 00 \\
1 \cdot 80\end{array}$ & $\begin{array}{r}208 \cdot 40 \\
41 \cdot 68\end{array}$ & $\begin{array}{r}+199 \cdot 40 \\
+39 \cdot 88\end{array}$ \\
\hline
\end{tabular}


The results expressed in the above table are represented graphically in the following curves:

$$
\text { Curve V., }
$$

showing the influence of boric acid and borax upon the nitrogen metabolism of an invalid child.

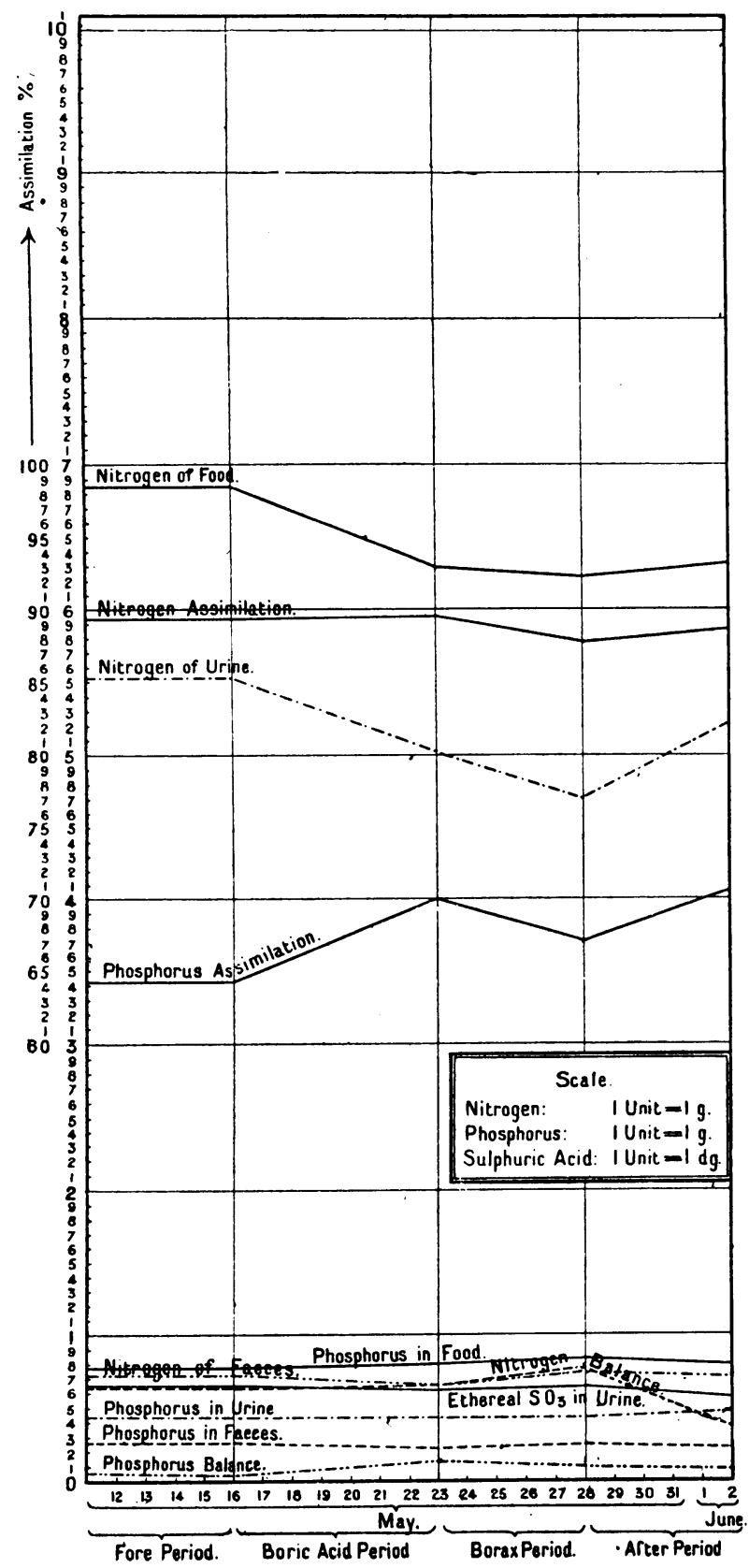




\section{Curve VI.,}

showing the influence of boric acid and borax upon the fat metabolism and the quantity of faeces and urine of an invalid child.

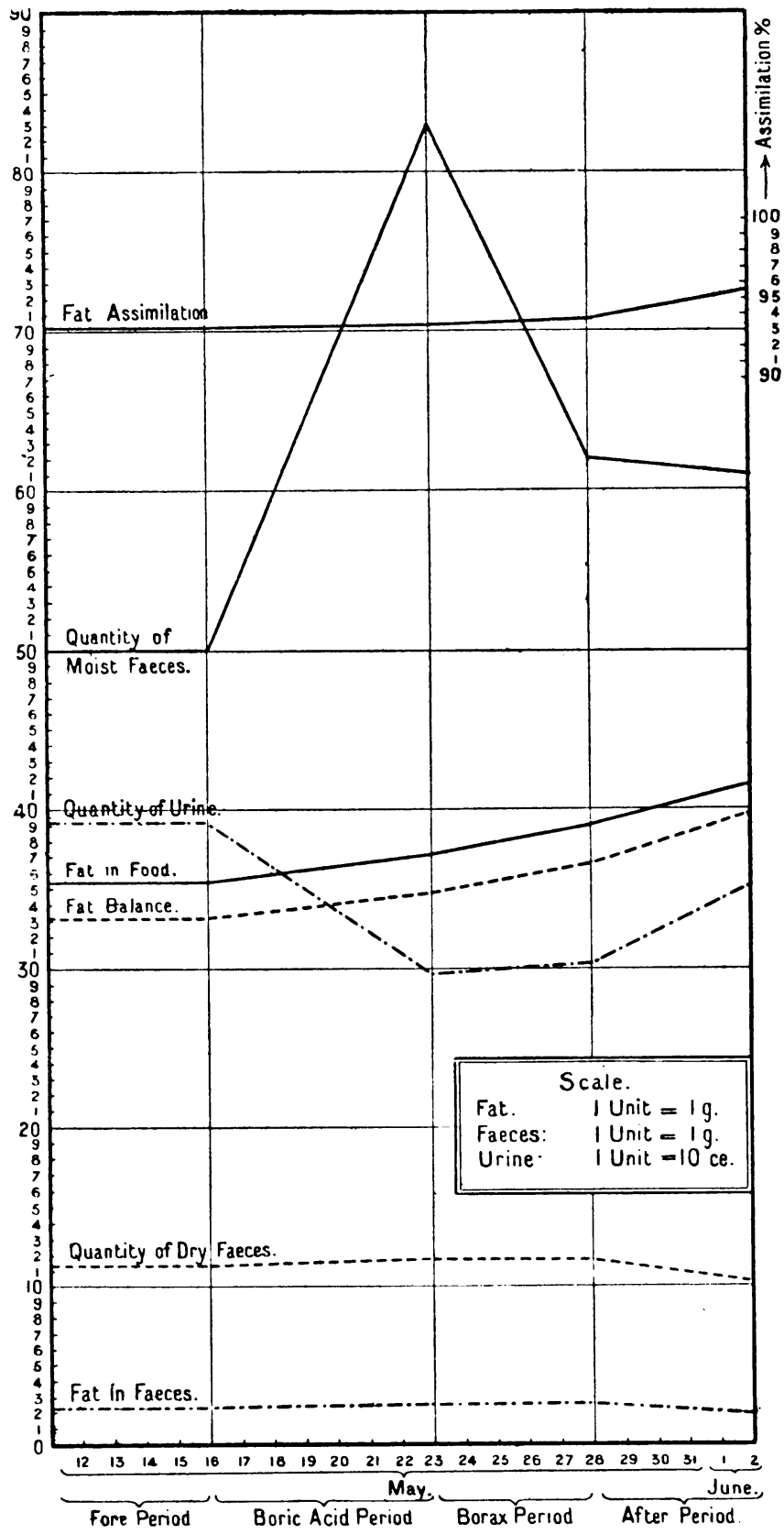


The uric acid excretion decreased somewhat during the boric acid period along with the decrease of nitrogen in food and the decreased nitrogen excretion in urine. During the borax period, however, we observed an increase in the amount of uric acid excreted, although the total nitrogen in the food and urine diminished. This seems to point to a specific uric acid solvent effect on the part of the borax and not to an increased uric acid formation, as in the after period the uric acid sank considerably below the fore period level.

The somewhat decreased quantity of total sulphuric acid excreted during the borax period, confirms the conclusion drawn from the decreased nitrogen and phosphorus, namely, that borax tends to slightly inhibit katabolism.

As in the former case, neither substance exerted an intestinal antiseptic action, borax probably by virtue of its alkalinity tending to increase the amount of ethereal sulphates eliminated.

Boric acid could be demonstrated in the urine in the first day of its administration, and was completely absent on the second day of the after period.

The body weight increased during both boric acid and borax period.

The results relevant to the observations made above are summarised in the following table:

Table IV a.

\begin{tabular}{|c|c|c|c|c|c|c|c|c|}
\hline & \begin{tabular}{|} 
Nitrogen \\
assimilation \\
$\%$ \%
\end{tabular} & $\begin{array}{l}\% \mathbf{N} \\
\text { of dry } \\
\text { faeces }\end{array}$ & $\begin{array}{l}\text { Phosphorus } \\
\text { assimilation } \\
\% \%\end{array}$ & $\begin{array}{l}\% \mathrm{P} . \\
\text { of dry } \\
\text { faeces }\end{array}$ & $\underset{0 \%}{\text { Fat }}$ & $\begin{array}{l}\% \text { fat } \\
\text { of dry } \\
\text { faeces }\end{array}$ & $\frac{\mathbf{A}^{*}}{\mathbf{B}}$ & $\frac{\mathrm{N}+}{\mathrm{SO}_{3}}$ \\
\hline Fore period & $89 \cdot 52$ & $6 \cdot 3$ & $64 \cdot 46$ & $2 \cdot 4$ & $93 \cdot 38$ & $20 \cdot 6$ & $15 \cdot 5$ & $5 \cdot 3$ \\
\hline Boric acid period & $89 \cdot 66$ & $5 \cdot 6$ & $70 \cdot 00$ & $2 \cdot 1$ & $93 \cdot 43$ & $20 \cdot 8$ & $13 \cdot 5$ & $5 \cdot 5$ \\
\hline Borax period & $87 \cdot 94$ & $6 \cdot 4$ & $67 \cdot 23$ & $2 \cdot 1$ & $93 \cdot 64$ & $21 \cdot 2$ & $12 \cdot 5$ & $5 \cdot 3$ \\
\hline After period & $88 \cdot 61$ & $7 \cdot 0$ & $69 \cdot 88$ & $2 \cdot 3$ & $95 \cdot 68$ & $17 \cdot 8$ & $15 \cdot 9$ & $5 \cdot 2$ \\
\hline
\end{tabular}

In all the three observations we estimated the amount of lecithin in the faeces during the normal and drug periods. The result of these investigations together with others will form the subject of a future paper, and we will restrict ourselves here to the simple statement that the excretion of lecithin with the faeces was diminished in each case during the borax periods. This observation, together with the fact of the improved phosphorus assimilation seems to point to a stimulating effect of this drug upon the pancreatic digestion, thus corroborating 
in vivo what has already been shown in vitro (compare Chittenden loc. cit.).

Before proceeding to draw our general conclusions we give for the sake of reference, in our diagram, the result of the three observations expressed graphically, in so far as regards the influence of boric acid and borax upon nitrogen, phosphorus, and fat assimilation and bodyweight.

\section{CuRve VII.,}

showing the influence of boric acid and borax upon the body weight, and upon the nitrogen, phosphorus, and fat assimilation of these children.

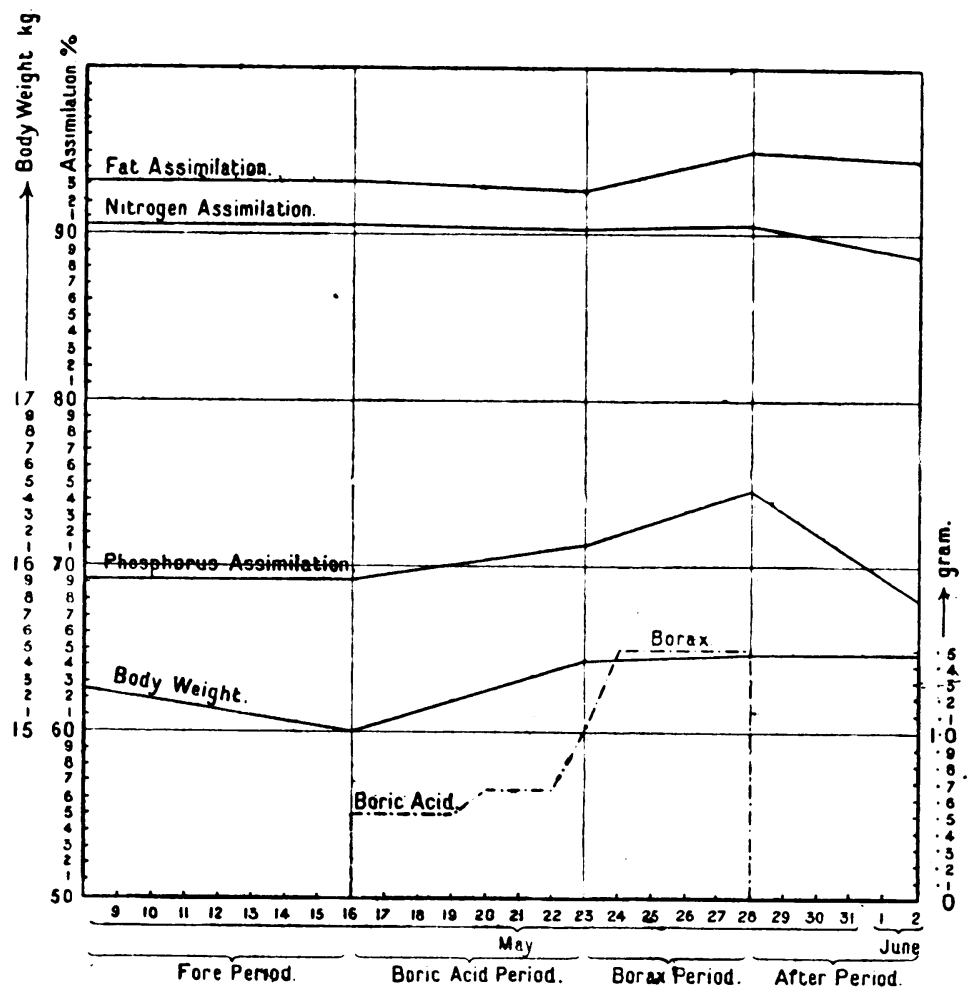

Child A. 
Curve VII.

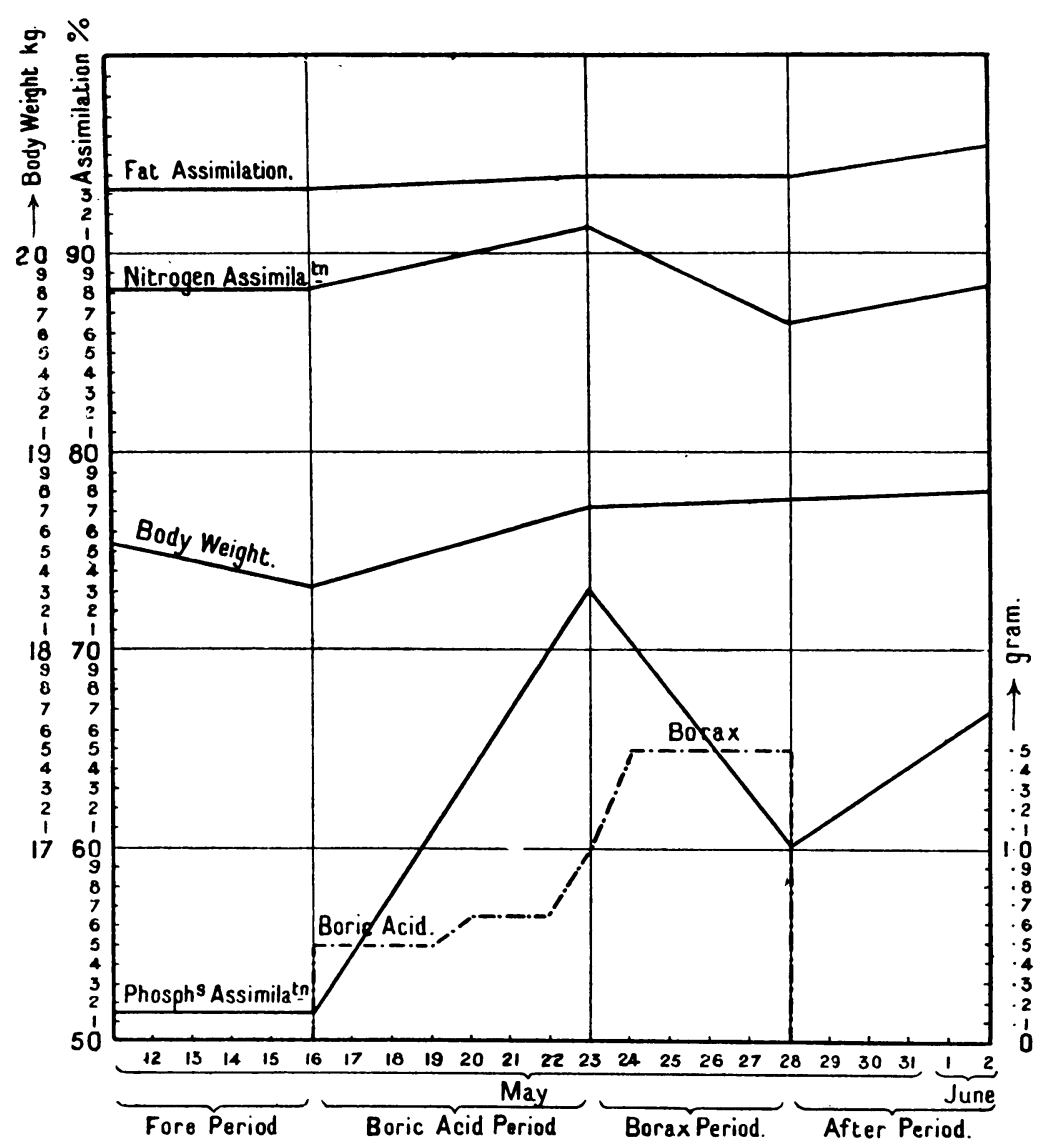

Child B. 
Curve VII.

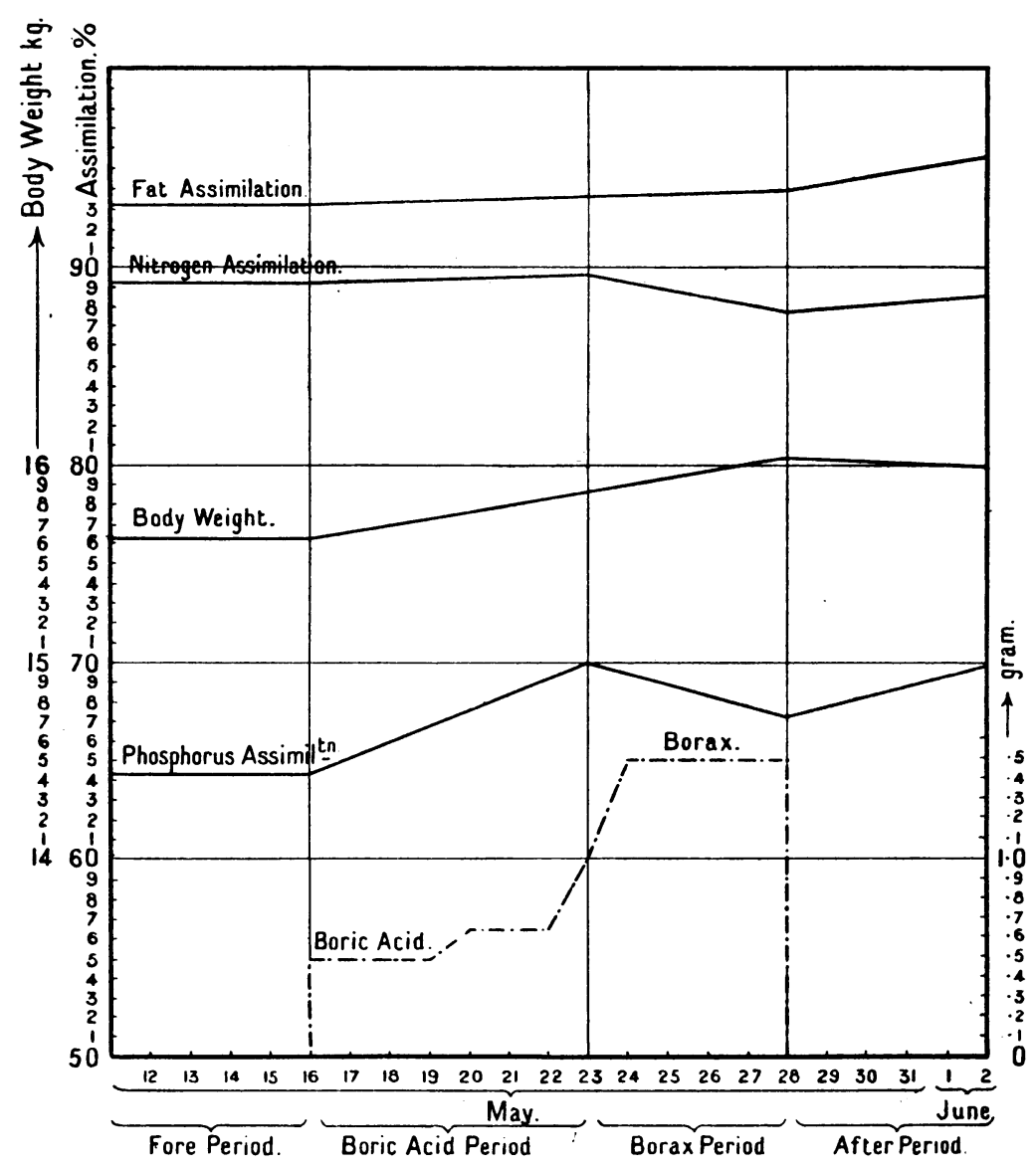

Child C. 


\section{General Conclusions.}

\section{Boric Acid.}

(1) Small doses up to 1 gramme per diem, continued for some time, exert in healthy or delicate children no influence upon proteid metabolism. The assimilation of the proteid food was improved in one healthy child (B).

(2) The phosphorus metabolism was unaffected in all cases. The assimilation of phosphorus was in all cases improved.

(3) The assimilation of fat was not affected.

(4) The body weight increased in all cases.

(5) The quantity of dry faeces was not affected. Their nitrogen and phosphorus percentage was slightly decreased.

(6) No inhibitory effect upon intestinal putrefaction could be demonstrated.

\section{Borax.}

(1) Continued doses of $1.5 \mathrm{~g}$. have no influence in healthy or delicate children upon proteid metabolism. The proteid assimilation was unaffected in healthy children, slightly depressed in the delicate child.

(2) The phosphorus metabolism was not affected in healthy or delicate children. The assimilation of phosphorus was improved in all cases, the improvement being least marked in the case of the delicate child.

(3) The fat assimilation was improved in the case of one healthy child, and unaffected in the case of the others.

(4) The body weight was increased in all cases; the increase was most marked in the case of the delicate child.

(5) The weight of dry faeces and their nitrogen and phosphorus percentage remained unaltered.

(6) Borax tended rather to increase intestinal putrefaction. 


\section{Boric Acid and Borax.}

(1) Both boric acid and borax were quickly eliminated, no cumulative action being therefore probable.

(2) Neither boric acid nor borax in any way affected the general health and well-being of the children.

If we compare these results with those obtained in the only previous complete observation made by Forster (loc. cit.) on the action of boric acid upon the general metabolism of one adult man, we find that they are only in accord in so far as in neither could any material effect upon the general health and metabolism be observed. In none of our three cases, however, could we confirm Forster's single observation that boric acid caused an increase in the quantity of faeces and in their nitrogen and phosphorus percentage. Further in contradistinction to Forster we were unable to find that boric acid exerted any inhibitory effect on intestinal putrefactive action.

If on the other hand we compare our results upon children with those obtained by Chittenden and Gies (loc. cit.) with similar doses of these substances upon the metabolism of dogs, it will be seen that in the essential points they agree.

King's College, London.

Jan. 1901. 